Collection SFN 8 (2007) 77-102

(c) EDP Sciences, Les Ulis

DOI: $10.1051 / \mathrm{sfn}: 2007008$

\title{
Magnétisme de surface et d'interface
}

\author{
F. Ott ${ }^{1}$ et M. Viret ${ }^{2}$
}

${ }^{1}$ Laboratoire Léon Brillouin, UMR 12 CEA/CNRS, CEA Saclay, 91191 Gif-sur-Yvette, France

${ }^{2}$ DRECAMISPEC, CEA Saclay, 91191 Gif-sur-Yvette, France

\section{INTRODUCTION}

Notre objectif est de caractériser des surfaces et des interfaces magnétiques à l'échelle nanométrique. Cela correspond à de très petits volumes de matière de l'ordre de quelques micro grammes. C'est pourquoi il est nécessaire de développer des techniques de diffusion de surface. L'utilisation de la réflexion de neutrons en incidence rasante augmente l'interaction des neutrons avec l'échantillon et rend de telles expériences faisables.

Il est possible de distinguer trois géométries de diffusion (voir Figure 1) : réflexion spéculaire, diffusion dans le plan d'incidence (diffusion hors spéculaire) et diffusion perpendiculairement au plan d'incidence. Ces différentes géométries de diffusion permettent de sonder différentes longueurs caractéristiques $\xi$ et directions sur la surface de l'échantillon. Dans ce chapitre nous allons nous focaliser uniquement sur la réflectivité spéculaire qui permet de sonder la structure le long de la profondeur dans le film $(3 \mathrm{~nm}<\xi<100 \mathrm{~nm})$. Nous présenterons le principe de la technique et des exemples d'applications sur différents systémes.

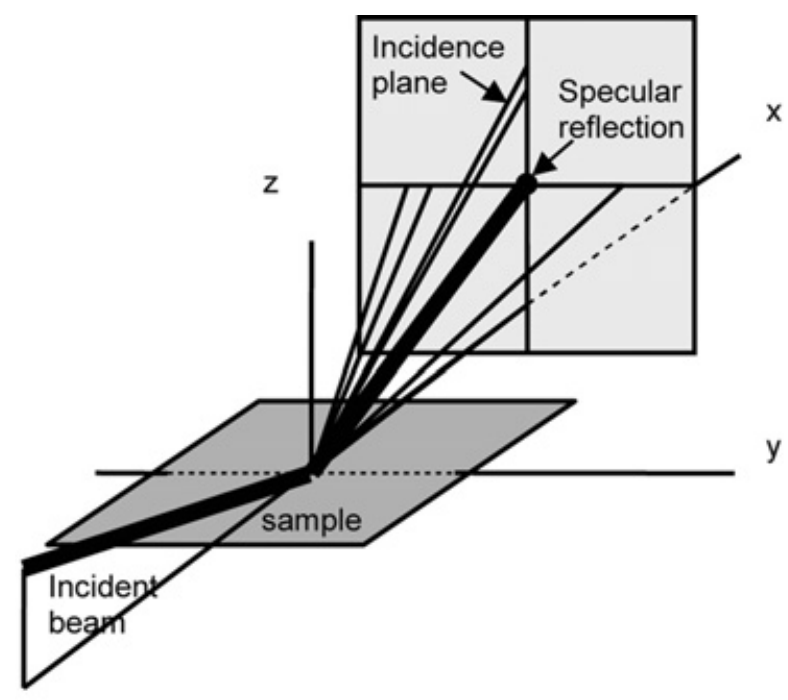

Specular reflection

$0.06<\mathrm{Q}_{\mathrm{z}}<3 \mathrm{~nm}^{-1}$

$3 \mathrm{~nm}<\xi<100 \mathrm{~nm}$

Incidence plane

$10^{-4}<\mathrm{Q}_{\mathrm{x}}<10^{-2} \mathrm{~nm}^{-1}$

$600 \mathrm{~nm}<\xi<60 \mu \mathrm{m}$

Plane perpendicular

$y \quad$ to the incidence plane

$10^{-4}<\mathrm{Q}_{\mathrm{y}}<3 \mathrm{~nm}^{-1}$

$3 \mathrm{~nm}<\xi<100 \mathrm{~nm}$

Figure 1. Les différentes géométries de diffusion de surface : (ligne continue) géométrie de réflectivité spéculaire, (surface avec des points) plan de diffusion hors spéculaire, correspondant au plan d'incidence, (surface hachurée) plan de diffusion perpendiculaire au plan d'incidence (GISANS). Ces différentes géométries de diffusion permettent de sonder une trés grande gamme de longueurs caractéristiques et de directions sur la surface de l'échantillon. 


\section{RÉFLECTIVITÉ SPÉCULAIRE}

\subsection{Réflexion des neutrons}

Soit $Q$ le vecteur de diffusion défini par :

$$
Q=k_{r}-k_{i}=\frac{4 \pi}{\lambda} \sin \theta
$$

Où $\lambda$ est la longueur d'onde du neutron, et $\theta$ est l'angle d'incidence du faisceau de neutrons sur la surface (voir Figure 2). Dans cette géométrie, l'angle de réflexion est égal à l'angle d'incidence ; le vecteur de diffusion $\boldsymbol{Q}$ est perpendiculaire à la surface de l'échantillon.

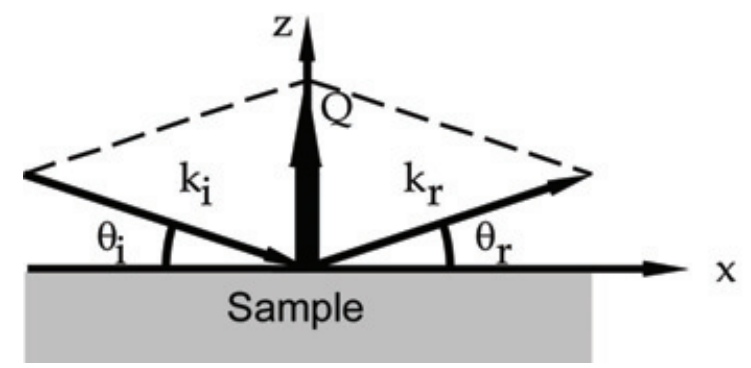

Figure 2. Géométrie de réflexion spéculaire. L'angle de réflexion est égal à l'angle d'incidence ; le vecteur de diffusion $\boldsymbol{Q}$ est perpendiculaire à la surface de l'échantillon.

Si on considère une surface, elle est composée d'atomes (voir Figure 3). Cependant, comme les angles d'incidence sont très petits, de l'ordre de quelques degrés, le vecteur de diffusion $\boldsymbol{Q}$ est de l'ordre de quelques $\mathrm{nm}^{-1}$ (comme déduit de l'Eq. 1). C'est-à-dire que l'on sonde des longueurs caractéristiques de l'ordre de quelques nanométres. Les détails atomiques de la structure sont invisibles. Il est possible d'appliquer l'approximation optique et de considérer le matériau comme un milieu continu. Nous introduisons la densité de longueur de diffusion (SLD Scattering Length Density) donnée par :

$$
b^{v o l}=\frac{1}{V} \sum_{i \in v o l} b_{i}
$$

Où $b_{i}$ est la longueur de diffusion de l'espéce $i$, et $V$ est le volume sur lequel la structure est moyennée (typiquement une cellule atomique.)

Dans une géométrie de réflectivité spéculaire, on peut faire l'hypothése que le systéme est invariant par translation le long de la surface de l'échantillon de telle sorte que les contributions de la diffusion proviennent uniquement de la structure le long de l'axe $(O z)$. La Figure 3 illustre la maniére dont une interface est modélisée dans une expérience de rèflectivitè. La longueur de diffusion varie avec la profondeur $z$ dans le film.

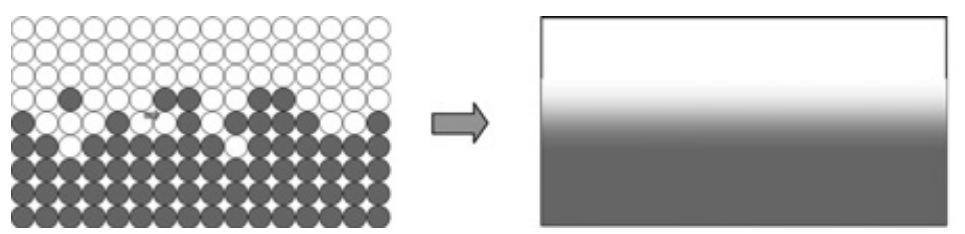

Figure 3. Interface entre deux surfaces. Dans l'approximation optique, l'interface est modèlisèe par un milieu continu de longueurs de diffusion. 


\subsection{Quelles informations obtient-on dans une mesure de réflectivité?}

Le potentiel d'interaction du neutron est donné par :

$$
V(z)=\frac{h^{2}}{2 \pi m_{n}} S L D(z)
$$

Où $S L D(z)$ est la densité de longueur de diffusion (Scattering Length Density SLD) le long de la direction $(O z)$. Soit $R(Q)$ l'intensité réfléchie en fonction du vecteur de diffusion. Dans l'approximation de Born, la réflectivité spéculaire mesure la transformée de Fourier du potentiel d'interaction $V(z)$ le long de la direction perpendiculaire à la surface de l'échantillon :

$$
R(Q) \propto F T(V(z))
$$

Cependant, le faisceau de neutrons peut être totalement réflèchi et alors l'approximation de Born n'est plus valide. Il est alors nécessaire de réaliser un calcul dynamique complet.

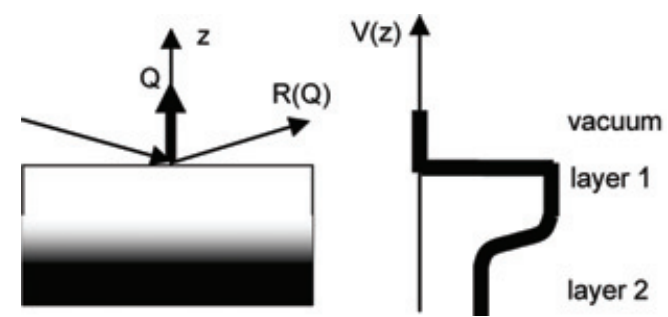

Figure 4. Réflexion sur un film mince déposé sur une surface. La réflectivité mesure la transformée de Fourier du potentiel d'interaction $V(z)$.

\subsection{Calcul de la réflectivité}

Le comportement du neutron est régi par l'équation de Schrödinger :

$$
\frac{-\hbar^{2}}{2 m_{n}} \Delta \varphi+V \varphi=E \varphi
$$

Où $\varphi$ est la fonction d'onde du neutron, $E$ est l'énergie cinétique du neutron et $V$ est le potentiel d'interaction (voir Eq. 3). Cette équation peut s'exprimer sous la forme d'une équation de propagation de Helmholtz :

$$
\Delta U+k^{2} U=0
$$

Où le vecteur d'onde propagation dans le milieu de potentiel $V$ est donné par :

$$
k^{2}=\frac{2 m_{n}}{\hbar^{2}}(E-V)
$$

Indice optique

Comme les neutrons suivent une équation de propagation classique, il est possible de définir un indice optique $n$ dans le milieu :

$$
n^{2}=\frac{k^{2}}{k_{0}^{2}}
$$

Où $k_{0}$ est le vecteur dans le vide. 
L'indice optique est donné par :

$$
n^{2}=1-\frac{V}{E}=1-\frac{\lambda^{2}}{\pi} S L D
$$

Qui peut être approximé avec une grande précision par :

$$
n \approx 1-\frac{\lambda^{2}}{2 \pi} S L D
$$

À une interface, la réflexion du neutron suit les lois de Snell classiques (voir Figure 5) :

$$
\cos \theta_{i}=n \cos \theta_{t r}
$$

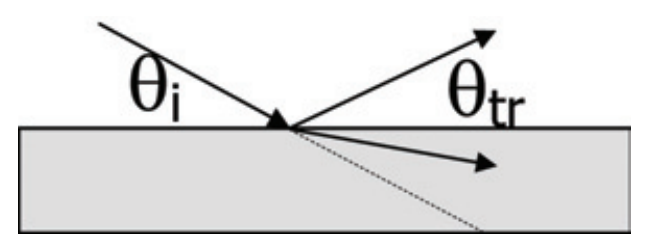

Figure 5. Réfraction d'un faisceau de neutrons à une interface.

Un point important est que l'indice optique est généralement plus petit que 1, cela signifie qu'en dessous d'un angle critique $\theta_{c}$ il $y$ a réflexion totale (définie par la condition $\theta_{t r}=0$ ). L'angle critique est donc donné par l'équation suivante :

$$
n=\cos \theta_{c}
$$

L'expression de l'angle critique est :

$$
\theta_{c}=\sqrt{\frac{S L D}{\pi} \lambda}
$$

Le vecteur d'onde critique est donné par :

$$
q_{c}=4 \sqrt{\pi S L D}
$$

Il est pratique d'exprimer l'indice optique sous la forme :

$$
n \approx 1-\delta=1-\frac{\lambda^{2}}{2 \pi} S L D
$$

Où $\delta$ est une petite déviation par rapport à 1 . Il est à noter que l'indice optique dépend de la longueur d'onde et donc la meilleure caractérisation d'un matériau est donnée par la $S L D$. La table 1 résume les indices optiques des matériaux les plus courants. 
Table 1. Indices optiques de quelques matériaux courants (donnés pour $\lambda=0.4 \mathrm{~nm}$ ).

\begin{tabular}{|l|l|l|}
\hline élément & $\delta\left(10^{-6}\right)$ & $\theta_{c}\left(^{\circ}\right)$ \\
\hline $\mathrm{Fe}$ & 20.45 & 0.36 \\
\hline $\mathrm{Co}$ & 5.7 & 0.19 \\
\hline $\mathrm{Ni}$ & 24 & 0.4 \\
\hline $\mathrm{Gd}$ & 5.0 & 0.18 \\
\hline $\mathrm{Si}$ & 5.3 & 0.19 \\
\hline $\mathrm{Ti}$ & -5 & - \\
\hline $\mathrm{Al}$ & 5.3 & 0.19 \\
\hline $\mathrm{SiO}_{2}$ & 10.1 & 0.26 \\
\hline
\end{tabular}

On notera que le nickel a l'indice optique le plus élevé de tous les matériaux. Une valeur utile à retenir est que l'angle critique de réflexion totale pour le nickel est de $0.1^{\circ} / \mathrm{A}^{\circ}$.

\section{Réflectivité sur des systèmes non magnétiques}

La réflectivité sur un substrat peut facilement être calculée. En dessous du vecteur d'onde critique $Q_{c}$, les neutrons sont totalement réfléchis : $R=1$. Approximativement au-dessus de $3 Q_{c}$, la réflectivité décroît comme $1 / Q^{4}$ (en suivant l'approximation de Born classique).

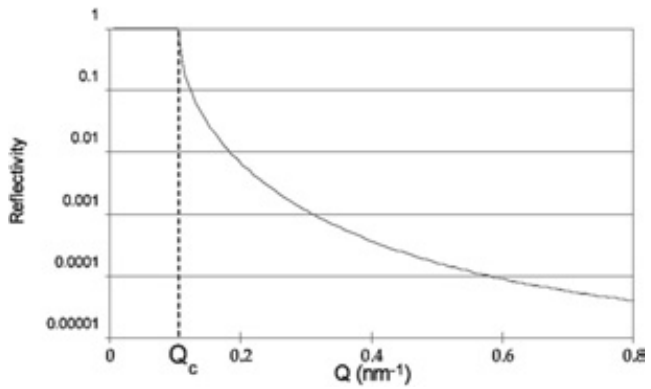

Figure 6. Réflectivité sur un milieu semi- infini, en dessous du vecteur d'onde critique $Q_{c}$, il $y$ a réflexion totale ; au-dessus de ce point, le signal décroît en $1 / Q^{4}$.

Dans le cas d'un film déposé sur un substrat, des oscillations apparaissent dans la courbe de réflectivité. Elles sont dues aux interférences constructives et destructives entre les ondes partiellement réfléchies

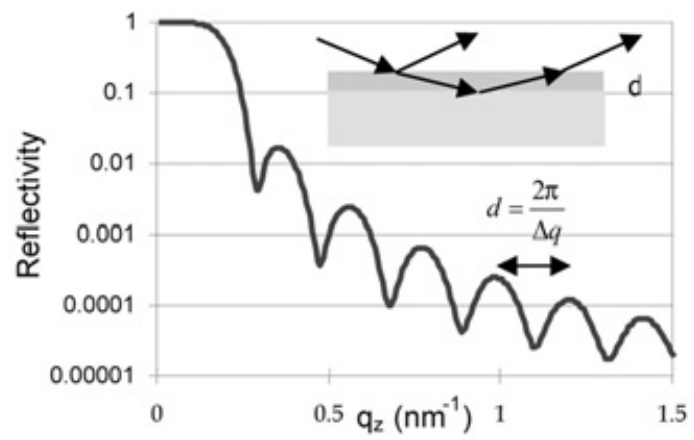

Figure 7. Réflectivité sur un film de cuivre (30 nm) déposé sur un substrat de silicium. 
sur les deux interfaces (voir Figure 7). La période des oscillations correspond à l'épaisseur de la couche : $d=2 \pi / \Delta q$. Ces oscillations sont souvent appelées franges de Kiessig.

Lorsque la multicouche est plus compliquée, la figure d'interférence est complexe et un calcul numérique est nécessaire. La Figure 8 illustre le cas d'une couche de cuivre couverte par une fine couche de protection de chrome. La couche de couverture introduit une modulation à longue distance au-dessus des franges d'interférences dues à l'épaisseur de la couche de cuivre.

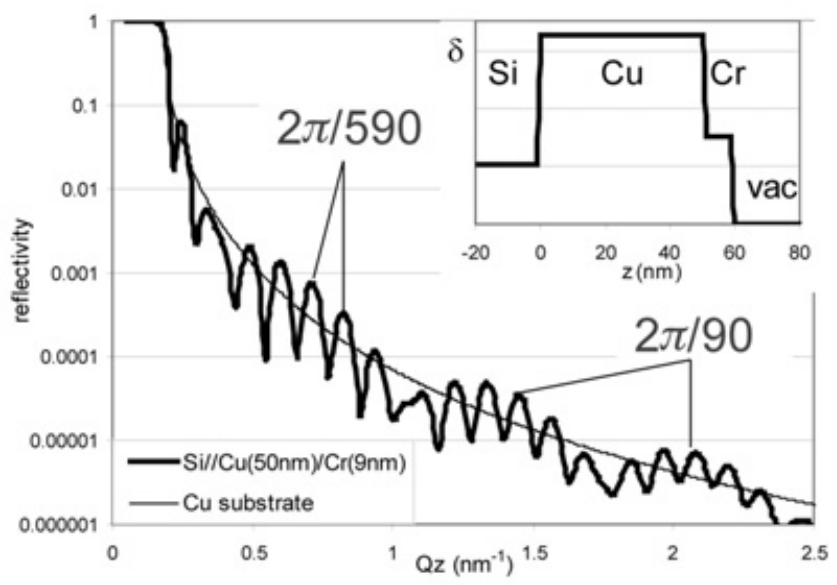

Figure 8. Réflectivité sur un milieu semi infini (substrat de cuivre) et sur un système multicouches $\mathrm{Si} / / \mathrm{Cu}(50 \mathrm{~nm}) /$ $\operatorname{Cr}(9 \mathrm{~nm})$. Les oscillations de courte période sont caractéristiques de l'épaisseur totale de la couche (59 $\mathrm{nm})$; la modulation à longue distance est caractéristique de la fine couche de couverture de chrome $(9 \mathrm{~nm})$. (Insert) profil d'indice optique en fonction de la profondeur dans le film.

\section{RÉFLECTIVITÉ DE NEUTRONS POLARISÉS}

\subsection{Interaction neutron magnétisme}

Au-delà de l'interaction nucléaire entre le neutron et les noyaux, il y a une interaction directe entre le spin du neutron et l'induction magnétique. Cette interaction est simplement donnée par l'interaction Zeeman :

$$
V_{M}(r)=-\vec{\mu}_{n} \cdot \vec{B}(r)
$$

Où $\mu_{n}$ est le moment magnétique du neutron et $B(r)$ est l'induction magnétique.

Dans un film mince continu, le champ magnétique dans la couche est donné par :

$$
B=\mu_{0}\left(H_{0}+(1-D) M\right)
$$

Où $H_{0}$ est le champ magnétique appliqué, $M$ est l'aimantation du film mince et $D$ est le facteur démagnétisant dans un film mince :

$$
(1-D) M=M_{/ /}
$$

Où $M_{/ /}$est la projection de l'aimantation dans le plan du film. Ceci est une limitation de la PNR : la technique est uniquement sensible à la composante planaire de l'aimantation. L'interaction du neutron avec la matière et donc donnée par :

$$
V=\frac{h^{2}}{2 \pi m} S L D-\mu_{n} \cdot \mu_{0} M-\mu_{n} \cdot B_{0}
$$


Afin de comparer les interactions nucléaires et magnétiques, il est possible d'exprimer le potentiel d'interaction sous la forme :

$$
V=\frac{h^{2}}{2 \pi m} \rho\left(b_{n}+b_{M}\right) \quad \text { avec } \quad b_{m}=\frac{2 \pi m}{h^{2}} \frac{\mu_{n} \mu_{0} M_{/ /}}{\rho}
$$

Où $\rho$ est la densité atomique.

La présente la comparaison entre les longueurs de diffusion nucléaire et magnétique pour les matériaux magnétiques les plus courants. On remarque immédiatement que les deux interactions sont du même ordre de grandeur. Dans le cas du fer, la longueur de diffusion magnétique est de l'ordre de 50\% de la longueur de diffusion nucléaire. Dans le cas du cobalt, la longueur de diffusion magnétique est deux fois plus importante que la longueur de diffusion nucléaire.

Table 2. Comparaison des longueurs de diffusion nucléaire et magnétique pour les matériaux magnétiques usuels.

\begin{tabular}{|l|l|l|}
\hline Éléments & $b_{n}(\mathrm{fm})$ & $b_{M}(\mathrm{fm})$ \\
\hline $\mathrm{Fe}$ & 9.45 & 5.4 \\
\hline $\mathrm{Co}$ & 2.49 & 4.5 \\
\hline $\mathrm{Ni}$ & 10.3 & 1.6 \\
\hline $\mathrm{Gd}$ & $6.5-\mathrm{i} 13.82$ & 18.83 \\
\hline $\mathrm{Si}$ & 4.15 & - \\
\hline $\mathrm{Ti}$ & -3.44 & - \\
\hline
\end{tabular}

\subsection{Réflectivité sur un système magnétique}

Dans la suite, nous appellerons "up" (resp. "down”), les neutrons dont la polarisation est parallèle (resp. antiparallèle) au champ magnétique appliqué qui définit l'axe de quantification. La fonction d'onde du neutron s'exprime sous la forme d'un spineur :

$$
\varphi=a|+\rangle+b|-\rangle=\left(\begin{array}{c}
a \\
b
\end{array}\right) \begin{aligned}
& |+\rangle \\
& |-\rangle
\end{aligned}
$$

Dans le cas d'un film mince magnétique, le système est biréfringent. L'indice optique dépend de la polarisation du neutron par rapport à l'aimantation. L'indice optique s'exprime comme :

$$
n^{ \pm} \approx 1-\delta \pm \delta_{M}=1-\frac{\lambda^{2}}{2 \pi} S L D \pm \frac{m \lambda^{2}}{h^{2}} \mu \cdot B
$$

Où $n^{+}$et l'indice optique pour les neutrons "up" et $n^{-}$l'indice optique pour les neutrons "down".

Dans une expérience classique de PNR, il est possible de mesurer quatre sections efficaces :

$$
R^{++}, R^{--}, R^{+-}, R^{-+}
$$

$R^{++}$correspond à l'amplitude réfléchie d'un faisceau de neutrons incident "up" et à la mesure de la fraction de neutron "up" après réflexion, c'est-à-dire sans spin-flip pendant la réflexion.

$R^{+-}$correspond à l'amplitude réfléchie d'un faisceau de neutrons incidents "up" et à la mesure de la fraction de neutrons "down" réfléchis, c'est-à-dire la fraction du faisceau qui a subi un spin-flip pendant la réflexion.

La matrice de transfert de l'échantillon est donnée par :

$$
\varphi_{\text {out }}=\left(\begin{array}{cc}
R^{++} & R^{+-} \\
R^{-+} & R^{--}
\end{array}\right) \varphi_{\text {in }}
$$

On peut distinguer deux cas extrêmes. 
Aimantation parallèle au champ magnétique appliqué

Pendant la réflexion, le neutron ne subit pas de spin-flip. Il reste dans son état propre. La matrice de transfert de l'échantillon est de la forme :

$$
\left(\begin{array}{cc}
R^{+} & 0 \\
0 & R^{-}
\end{array}\right)
$$

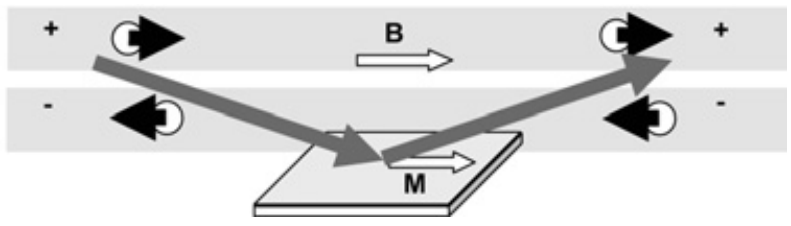

Figure 9. Dans le cas d'une aimantation parallèle au champ magnétique appliqué, il est possible de mesurer 2 sections efficaces différentes $R^{++}$et $R^{--}$.

Pour un faisceau polarisé "up" (resp. "down”), $\varphi_{\text {in }}=1|+\rangle\left(\right.$ resp. $\left.\varphi_{\text {in }}=1|-\rangle\right)$

$$
\begin{gathered}
\varphi_{\text {out }}=\left[\begin{array}{c}
R^{+} \\
0
\end{array}\right]=\left(\begin{array}{cc}
R^{+} & 0 \\
0 & R^{-}
\end{array}\right)\left[\begin{array}{l}
1 \\
0
\end{array}\right] \begin{array}{l}
|+\rangle \\
|-\rangle
\end{array} \text { et } \\
\varphi_{\text {out }}=\left[\begin{array}{c}
0 \\
R^{-}
\end{array}\right]=\left(\begin{array}{cc}
R^{+} & 0 \\
0 & R^{-}
\end{array}\right)\left[\begin{array}{l}
0 \\
1
\end{array}\right] \begin{array}{l}
|+\rangle \\
|-\rangle
\end{array}
\end{gathered}
$$

Les amplitudes de réflexion sont données par :

$$
r^{++}=\frac{k_{\perp 0}-k_{\perp s}^{+}}{k_{\perp 0}+k_{\perp s}^{+}} \quad \text { and } \quad r^{--}=\frac{k_{\perp 0}-k_{\perp s}^{-}}{k_{\perp 0}+k_{\perp s}^{-}}
$$

Les intensités réfléchies sont données par :

$$
R^{+}=\left|r^{++}\right|^{2} \quad \text { and } \quad R^{-}=\left|r^{--}\right|^{2}
$$

L'angle critique pour les neutrons up et down est donné par :

$$
n^{+}=\cos \theta_{c}^{+} \quad \text { et } \quad n^{-}=\cos \theta_{c}^{-}
$$
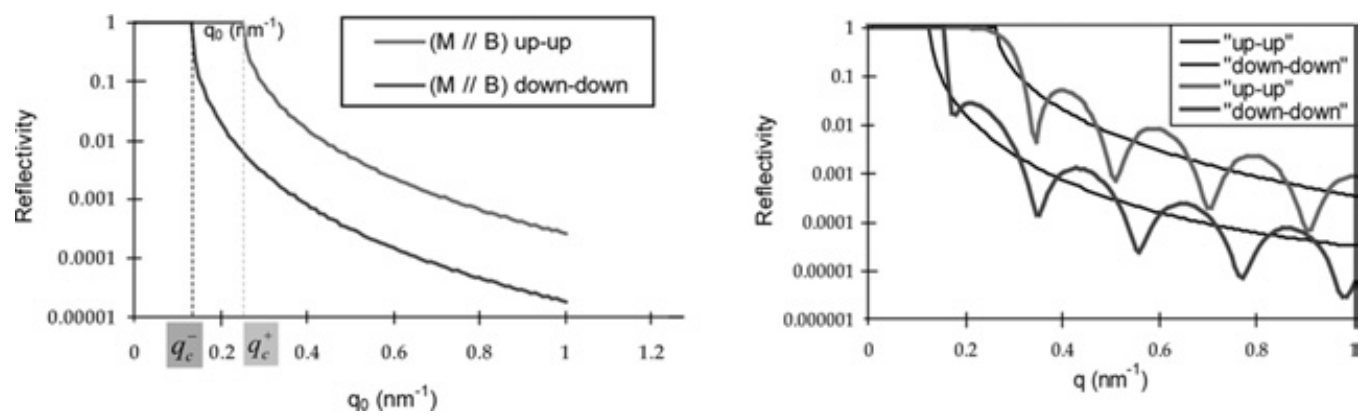

Figure 10. (a) Réflexion sur un milieu magnétique semi-infini ; (b) réflexion sur un film mince magnétique (Fe $(30 \mathrm{~nm})$ sur saphir). Les oscillations sont directement reliées à l'épaisseur du film de fer. La séparation entre les courbes est directement liée à l'aimantation. 
La réflexion sur un milieu magnétique semi-infini est très proche du cas magnétique. La seule différence est que les courbes "up" et "down" sont distinctes. La différence est directement reliée à l'amplitude de l'aimantation.

\section{Aimantation perpendiculaire au champ magnétique appliqué}

Pendant la réflexion, le neutron va subir un spin-flip partiel, il précesse autour de l'aimantation pendant la réflexion. La matrice de transfert de l'échantillon a la forme générale :

$$
\left(\begin{array}{cc}
R^{++} & R^{+-} \\
R^{-+} & R^{--}
\end{array}\right)
$$

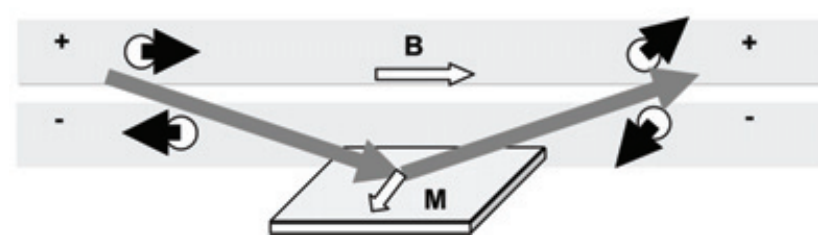

Figure 11. Dans le cas d'une aimantation perpendiculaire au champ magnétique appliqué, les neutrons ne restent pas dans leur état propre. Il est possible de mesurer quatre sections efficaces de diffusion $R^{++}, R^{--}, R^{+-}, R^{-+}$.

Pour un faisceau polarisé "up" (resp. "down”), $\varphi_{\text {in }}=1|+\rangle$ (resp. $\varphi_{\text {in }}=1|-\rangle$ )

$$
\begin{aligned}
\varphi_{\text {out }}=\left[\begin{array}{l}
R^{++} \\
R^{+-}
\end{array}\right] & =\left(\begin{array}{ll}
R^{++} & R^{+-} \\
R^{-+} & R^{--}
\end{array}\right)\left[\begin{array}{l}
1 \\
0
\end{array}\right] \begin{array}{ll}
|+\rangle & \text { et } \\
|-\rangle
\end{array} \\
\varphi_{\text {out }} & =\left[\begin{array}{l}
R^{-+} \\
R^{--}
\end{array}\right]=\left(\begin{array}{ll}
R^{++} & R^{+-} \\
R^{-+} & R^{--}
\end{array}\right)\left[\begin{array}{l}
0 \\
1
\end{array}\right] \begin{array}{l}
|+\rangle \\
|-\rangle
\end{array}
\end{aligned}
$$

L'intensité de spin-flip réfléchie est donnée par :

$$
r^{+-}=\frac{1}{2} \frac{k_{\perp 0}\left(k_{\perp s}^{+}-k_{\perp s}^{-}\right)}{\left(k_{\perp 0}^{+}+k_{\perp s}^{-}\right)\left(k_{\perp 0}^{+}+k_{\perp s}^{-}\right)}
$$

Dans le cas où l'aimantation est strictement perpendiculaire au champ magnétique appliqué, les intensités réfléchies "up-up" et "down-down" sont égales et sont la somme de trois termes :

$$
R^{++}=R^{--}=\left|r^{2}\right|=\frac{1}{4}\left|r^{++}\right|^{2}+\frac{1}{4}\left|r^{--}\right|^{2}+\frac{1}{2} \operatorname{Re}\left(r^{++} \times r^{++}\right)
$$

Les deux premiers termes correspondent aux intensités de non spin-flip dans le cas d'une aimantation alignée avec le champ magnétique extérieur; elles sont pondérées par un coefficient $\frac{1}{4}$. Ces deux termes introduisent 2 discontinuités aux positions $q_{c}^{+}$et $q_{c}^{-}$dans les courbes de réflectivité (voir Figure 12). Un terme d'interférence s'ajoute à cela, dont l'expression n'est pas triviale. Il ne change pas qualitativement la forme des courbes.

L'intensité spin-flip est donnée par :

$$
R^{+-}=R^{-+}=\left|r^{+-}\right|^{2}=\frac{1}{4}\left|\frac{k_{\perp 0}\left(k_{\perp s}^{+}-k_{\perp s}^{-}\right)}{\left(k_{\perp 0}^{+}+k_{\perp s}^{-}\right)\left(k_{\perp 0}^{+}+k_{\perp s}^{-}\right)}\right|^{2}
$$

La forme caractéristique du signal de spin-flip (voir Figure 12) est donnée par le terme $\left|k_{\perp s}^{+}-k_{\perp s}^{-}\right|^{2}$. 

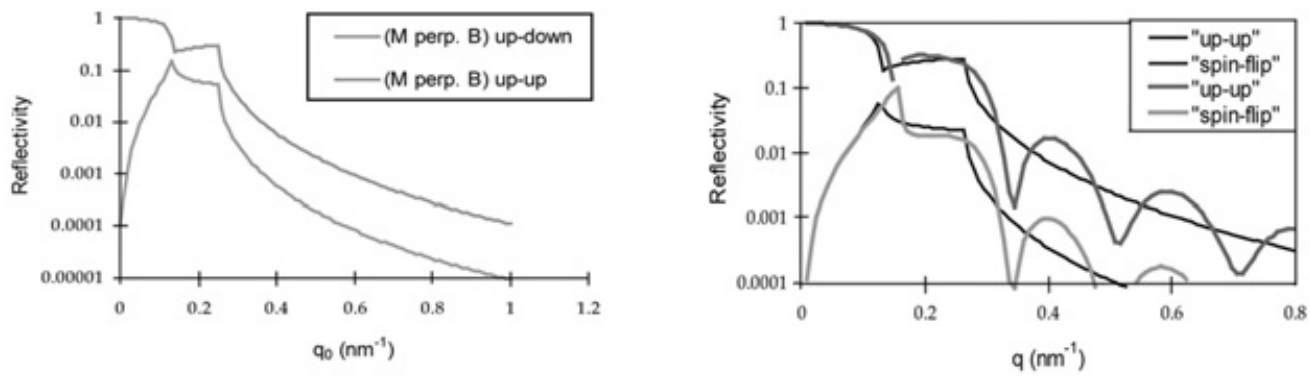

Figure 12. (a) Réflexion sur un milieu magnétique semi-infini où le champ guide est perpendiculaire à l'aimantation ; (b) réflexion sur un film mince magnétique $(\mathrm{Fe}(30 \mathrm{~nm})$ sur saphir) : des modulations apparaissent.

Dans une image simple, on peut résumer en disant que les sections efficaces $R^{++}$et $R^{--}$donnent des informations sur les composantes de l'aimantation le long du champ magnétique appliqué. Les sections efficaces de spin-flip $R^{+-}=R^{-+}$donnent des informations sur les composantes de l'aimantation perpendiculaire au champ magnétique appliqué. De manière plus générale, la PNR permet d'accéder à l'amplitude et à la direction de l'aimantation dans chaque couche d'un système multicouches. Cela permet de déterminer des profils d'aimantation en profondeur, de réaliser une mesure absolue de l'aimantation en $\mu B$ par f.u. (somme du moment orbital et de spin). La résolution est de l'ordre de $0.1 \mu B$. Comme il s'agit d'une technique de surface, on n'est pas sensible au para- ou dia-magnétisme du substrat. Il n'y a pas d'absorption, pas de paramètres phénoménologiques, la normalisation est absolue.

\section{EFFETS DE RUGOSITÉ}

En réalité, les systèmes étudiés sont toujours imparfaits. Les couches sont composées d'atomes et les interfaces ne sont jamais parfaites. Il est nécessaire de distinguer deux échelles de taille qui sont d'une importance cruciale. La première caractérise les fluctuations de hauteur de l'interface :

$$
\sigma=\sqrt{\left\langle z^{2}\right\rangle_{\text {surface }}}
$$

La seconde longueur caractéristique est donnée par $\xi$ qui caractérise les fluctuations planaires.

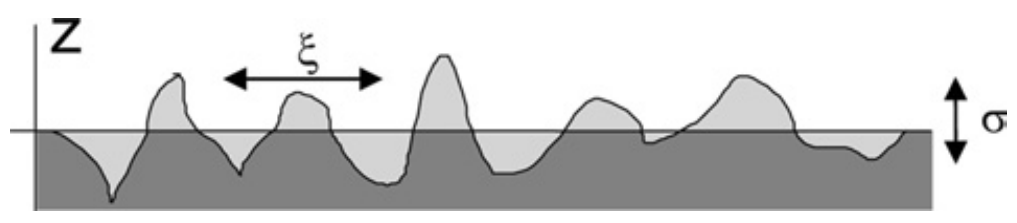

Figure 13. Interface entre deux matériaux n'est jamais parfaite. La perturbation par rapport à l'interface parfaite et représentée en gris clair.

En pratique, on peut distinguer quatre types de rugosité :

- la rugosité à l'échelle atomique : elle correspond à l'inter diffusion des atomes à l'interface, typiquement la rugosité $\sigma$ est de l'ordre de $0.5-1 \mathrm{~nm}$ et $\xi<100 \mathrm{~nm}$. Dans ce cas, la réflectivité spéculaire ne peut pas voir ces détails et le système peut être approximé par un profil mou (voir Figure 14(a)). 
- Rugosité intermédiaire : $\xi$ de $0.1 \mu \mathrm{m}$ à $50 \mu \mathrm{m}$ et $\sigma \sim 1 \mathrm{~nm}$.

Dans ce cas, une partie du signal diffusé par la surface n'est pas diffusée dans la direction spéculaire. Le signal de réflectivité spéculaire doit être corrigé par un facteur d'atténuation appelée facteur de Névot-Croce $e^{-\sigma^{2} q^{2}}$. Le signal diffusé hors de la direction spéculaire peut aussi être mesuré et caractérisé. Il donne des informations détaillées sur la structure planaire aux interfaces dans le film. Ce thème sera traité dans le chapitre sur la diffusion hors spéculaire (E. Kentzinger).

- une rugosité à grande échelle : $\xi>100 \mu \mathrm{m}$

Ces fluctuations correspondent habituellement à un substrat non plan (ou courbure de l'échantillon). Dans ce cas, le moyen le plus simple de traiter les données est de considérer que la divergence incidente ou la résolution en longueur d'onde sont augmentées. Il faut éviter cette situation car l'information de réflectivité est alors fortement altérée.

- Inhomogénéité de l'épaisseur de la couche

(a)

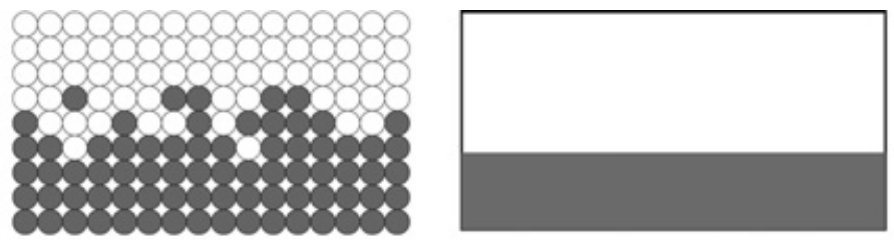

(b)

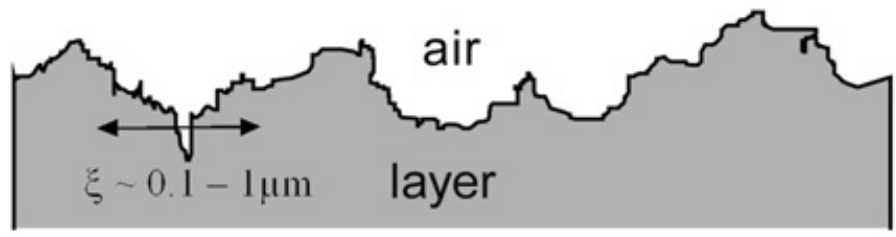

(c)

substrate

(d)

substrate

Figure 14. (a) Inter diffusion atomique entre deux couches ; les détails ne peuvent pas être observés dans une géométrie de réflectivité. (b) Rugosité à l'échelle micrométrique. (c) Courbure de l'échantillon. (d) Fluctuations de l'épaisseur de la couche.

Les différents types de rugosité sont comparés sur la Figure 15. Dans le cas de l'inter diffusion atomique (rouge), la forme du signal est très proche du système parfait à l'exception de l'épaisseur effective de la couche supérieure qui est plus faible et qui conduit à un décalage des oscillations. Dans le cas d'une interface rugueuse, des neutrons sont diffusés hors de la direction spéculaire et le signal décroît plus vite à grand vecteur de diffusion $Q$. Il faut appliquer le facteur de Névot-Croce pour rendre compte de cet effet. 

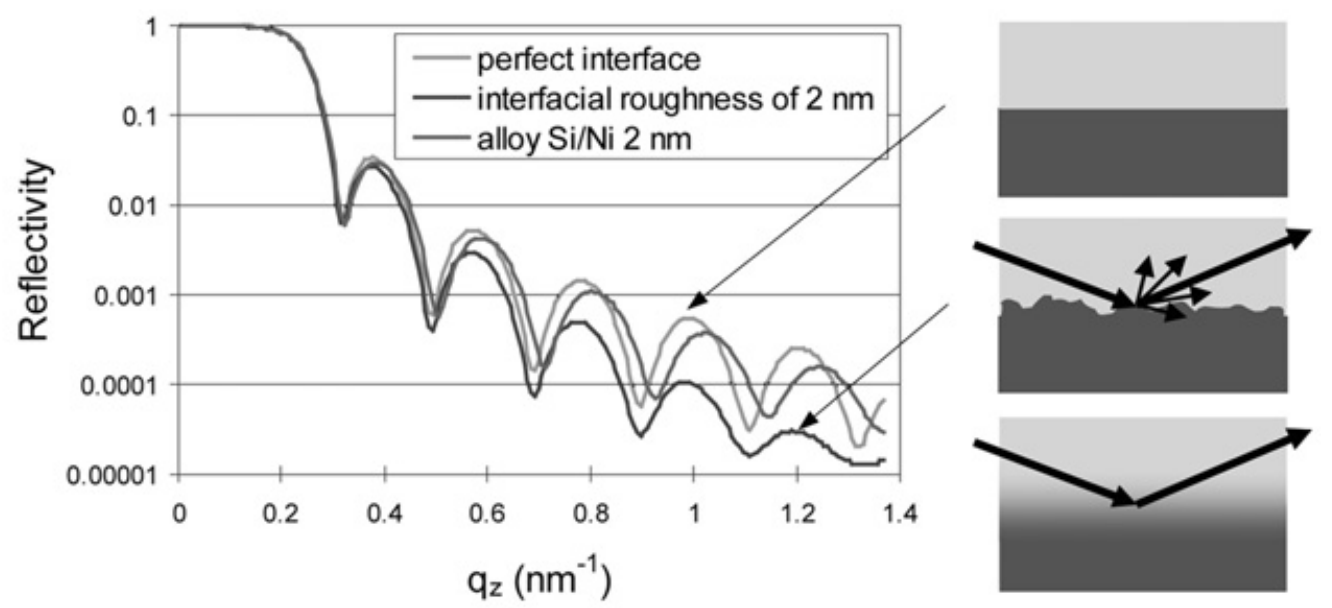

Figure 15. Influence de la rugosité sur la réflectivité. (a) interface parfaite. (b) interface rugueuse, des neutrons sont diffusés hors de la direction spéculaire et le signal décroît plus vite aux grandes valeurs de $Q$. (c) interface douce due à une diffusion atomique, les oscillations sont moins marquées car le contraste d'indice est plus faible.

\section{RÉALISATIONS DES EXPÉRIENCES-CONTRAINTES}

\section{Taille des échantillons-temps de mesure}

Il faut garder à l'esprit que la plupart des réflectomètres sont optimisés pour réaliser des expériences sur des échantillons ayant des surfaces de l'ordre de $10 \mathrm{~cm}^{2}$. Dans le cas des réflectomètres optimisés avec des systèmes de focalisation (comme le réflectomètre PRISM au LLB), il est possible de réaliser des expériences sur des échantillons dont la surface est de l'ordre de $1 \mathrm{~cm}^{2}$. Ces limitations sur la surface impliquent que les échantillons étudiés doivent avoir une très bonne homogénéité sur une grande surface : l'épaisseur des couches doit être homogène et les substrats doivent être plats sur toute la surface de l'échantillon. Si cela n'est pas le cas, seules des moyennes sur la surface de l'échantillon seront mesurées et l'information qui pourra être obtenue sur l'échantillon sera limitée. Le signal de réflectivité chute trés vite en fonction du vecteur d'onde. Pour une interface parfaite, à grandes valeurs de $Q$, la réflectivité est proportionnelle à $\frac{1}{Q^{4}}$. Des valeurs de $Q$ de l'ordre de $2-3 \mathrm{~nm}^{-1}$ correspondent à des réflectivités de l'ordre de $10^{-6}$ et nécessitent des mesures de l'ordre de deux à quatre heures.

\section{Environnements échantillons}

L'absorption des neutrons est négligeable dans la plupart des matériaux. La longueur de pénétration typique de matériaux tels que le silicium ou l'aluminium et de l'ordre de $50 \mathrm{~mm}$ (elle dépend de la longueur d'onde). Cela rend facile la mise en place d'environnements échantillon complexes : cryoaimant, champ magnétique, fours, cellules pour les liquides.

\section{Analyse des données expérimentales}

Les courbes de réflectivité ne peuvent pas être inversées directement. Il est souvent possible de construire une famille de profils de longueur de diffusion qui fournissent la même courbe de réflectivité. Cela est dû au fait que seule l'intensité est mesurée et non pas la phase de la réflectivité. L'analyse des données expérimentales est faite en ajustant les différents paramètres impliqués dans le problème jusqu'à ce que 
le meilleur fit au moindre carré soit obtenu. La principale source d'incertitude est liée au manque d'intensité à grands vecteurs de diffusion et à la rugosité de l'échantillon. Dans le cas de systèmes magnétiques, la composition et l'épaisseur des différentes couches sont habituellement assez bien connues. Il n'est alors nécessaire de fitter que la rugosité et les amplitudes des moments magnétiques. Il est en général très utile d'avoir des informations extérieures provenant de réflectométrie $X$, d'ellipsométrie ou de mesures magnétiques.

\section{PROPRIÉTÉS MAGNÉTIQUES DES SURFACES ET DES INTERFACES}

\subsection{Ferromagnétisme}

Il existe essentiellement deux types de ferromagnétisme.

- Magnétisme d'électrons localisés

Il est le plus souvent observé dans les terres rares et les composés ioniques. Un des exemples de couplage est le couplage dit de super-échange dans lequel les atomes magnétiques sont couplés magnétiquement à travers les orbitales de l'oxygène qui séparent les ions magnétiques.

\section{- Magnétisme de bande}

Ce type de magnétisme se retrouve essentiellement pour les métaux de transition (Fe, $\mathrm{Co}, \mathrm{Ni}$ ). L'apparition d'un état ferromagnétique est lié à une compétition entre énergie cinétique et couplage d'échange magnétique. Le critère de Stoner $J . D\left(E_{F}\right)>1$ définit la condition pour laquelle il $y$ a apparition d'un état ferromagnétique rémanent ( $J$ est le couplage d'échange magnétique, $D\left(E_{F}\right)$ est la densité d'état au niveau de Fermi).

\subsection{Effets de surface}

Au niveau des surfaces et des interfaces, en raison du nombre réduit de voisins, des frustrations apparaissent. Les liaisons sont différentes, les bandes de conduction sont plus étroites. Une plus grande densité d'états est favorable au critère de Stoner. Dans le cas de clusters de Rh et de Pt, cela donne naissance à des moments magnétiques dans ces matériaux. Dans le cas de systèmes confinés, les effets de température sont amplifiés (en général $T_{c}$ diminue).

(a)

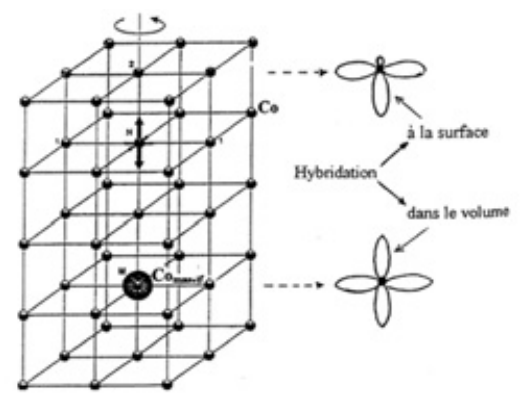

(b)

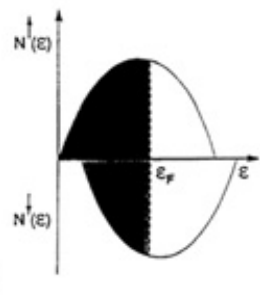

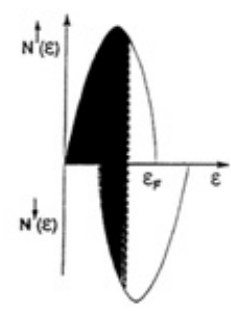

Figure 16. (a) Perte de symétrie de l'environnement à une surface. (b) modification de la densité d'état à une surface. 


\section{EXEMPLES DE SYSTÈMES}

Dans ce chapitre, nous allons illustrer les possibilités offertes par la technique de réflectivité de neutrons polarisés. Cette technique peut être appliquée à des systèmes variés :

- films minces : couches d'oxydes (magnétite, manganites)

- multicouches (systèmes à magnéto-résistance géante, GMR)

- super-réseaux : de terres rares (Y, Dy, Er, Tb), semi-conducteurs magnétiques (GaMnAs).

\subsection{Monochromateur multicouches}

Les technologies de films minces permettent de déposer des couches très minces sur des substrats. Il est ainsi possible de produire des cristaux 1D artificiels avec des périodicités aussi faibles que 5 $\mathrm{nm}$ (voir Figure 17). Cela permet notamment de produire des systèmes qui se comportent comme des monochromateurs.
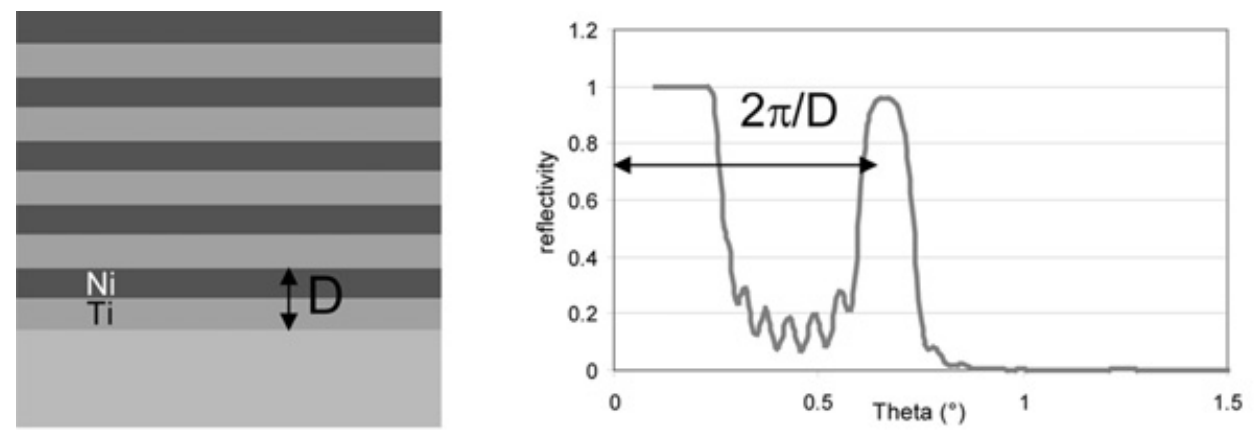

Figure 17. (a) Multicouches $[\mathrm{Ni}(5 \mathrm{~nm}) / \mathrm{Ti}(5 \mathrm{~nm})]_{10}$ déposées sur du verre. (b) réflectivité de la multicouche. En dessous de l'angle critique, il $y$ a réflexion totale; autour de $\theta=0.7^{\circ}$ il $y$ a un pic de diffraction correspondant à l'épaisseur de la bicouche D. La réflectivité peut être très élevée (proche de 95\%).

De telles structures sont maintenant fabriquées de manière routinière sur de très grandes surfaces (de l'ordre du mètre carré) pour produire des monochromateurs de neutrons. La Figure 18 montre la réflectivité d'un monochromateur produit par Swiss Neutronics. La période de la bicouche est de $9 \mathrm{~nm}$. La réflectivité de ce monochromateur atteint $80 \%$. Il est possible d'ajuster la bande de monochromatisation en ajustant la période des bicouches.
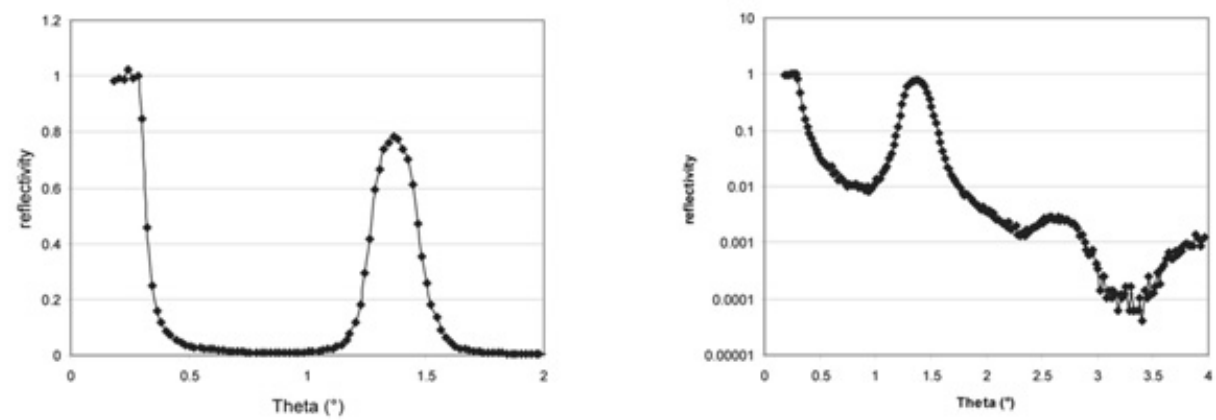

Figure 18. Réflectivité d'un monochromateur produit par Swiss Neutronics (gauche) échelle linéaire ; (droite) échelle logarithmique, les harmoniques de diffraction peuvent être mesurés. 
Les mesures dans l'espace direct par TEM montrent la qualité des interfaces dans ces multicouches métalliques.

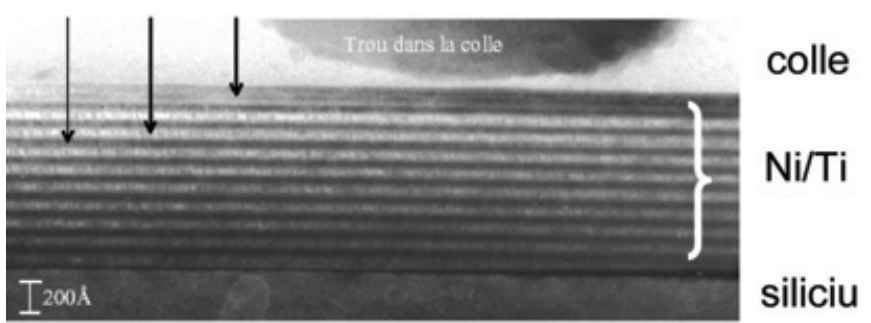

Figure 19. Image HRTEM dans l'espace direct d'un système multicouche.

\subsection{Polariseur de neutrons}

Il est possible d'introduire des matériaux magnétiques dans les systèmes multicouches.

Nota: dans l'exemple précédent, des éléments tels que le carbone sont ajoutés dans le nickel de telle sorte qu'il n'est pas magnétique.

Afin de produire un élément optique efficace, un indice optique élevé est nécessaire. Afin de maximiser le contraste magnétique, les alliages de Fe ou FeCo sont utilisés. L'espaceur est un matériau proche du silicium afin que son indice optique corresponde à l'indice optique $n^{-}$de la couche magnétique. Quand les neutrons sont polarisés « up », ils voient une forte modulation de l'indice optique (voir Figure 20). Quand les neutrons sont polarisés « down », ils voient un potentiel d'interaction lisse et il $n^{\prime} y$ a pas de diffraction. Un tel dispositif se comporte comme un monochromateur polarisant.

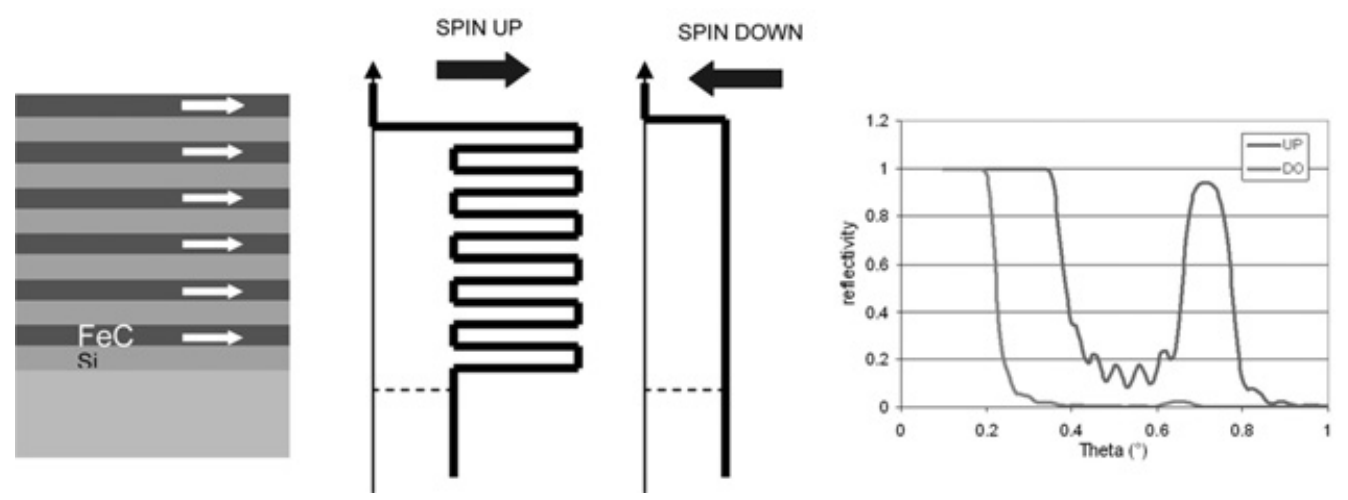

Figure 20. (a) Multicouches $[\mathrm{FeCo} / \mathrm{Si}]_{10}$ déposées sur verre. (b) Potentiel d'interaction en fonction de la polarisation. (c) Réflectivité de la multicouche.

Comme l'absorption des neutrons est très faible, il est possible d'empiler les multicouches de période variable afin de superposer une série de pics de diffraction. Il est ainsi possible de remplir la zone entre la réflexion totale et une valeur $Q_{\max }$ et d'obtenir un miroir d'indice optique artificiel très élevé. La valeur $Q_{\max }$ est limitée par les plus petites couches qu'il est possible de déposer (actuellement de 
l'ordre de $5 \mathrm{~nm}$ ). Cela permet de produire des miroirs qui ont un indice optique artificiel qui est 6 fois plus élevé que celui du nickel.

La Figure 21 montre la réflectivité d'un vrai polariseur (produit par l'ILL). En dessous de $Q=0.15 \mathrm{~nm}^{-1}$, les deux états de spin se réfléchissent. Au-dessus de $0.15 \mathrm{~nm}^{-1}$, l'état de spin « down » n'est pas réfléchi ; jusqu'à $0.6 \mathrm{~nm}^{-1}$, l'état de spin « up » est réfléchi. La Figure 21(b) montre le rapport de flipping up/down, il atteint des valeurs supérieures à 100. Cela correspond à une polarisation du faisceau supérieure à $99.3 \%$.
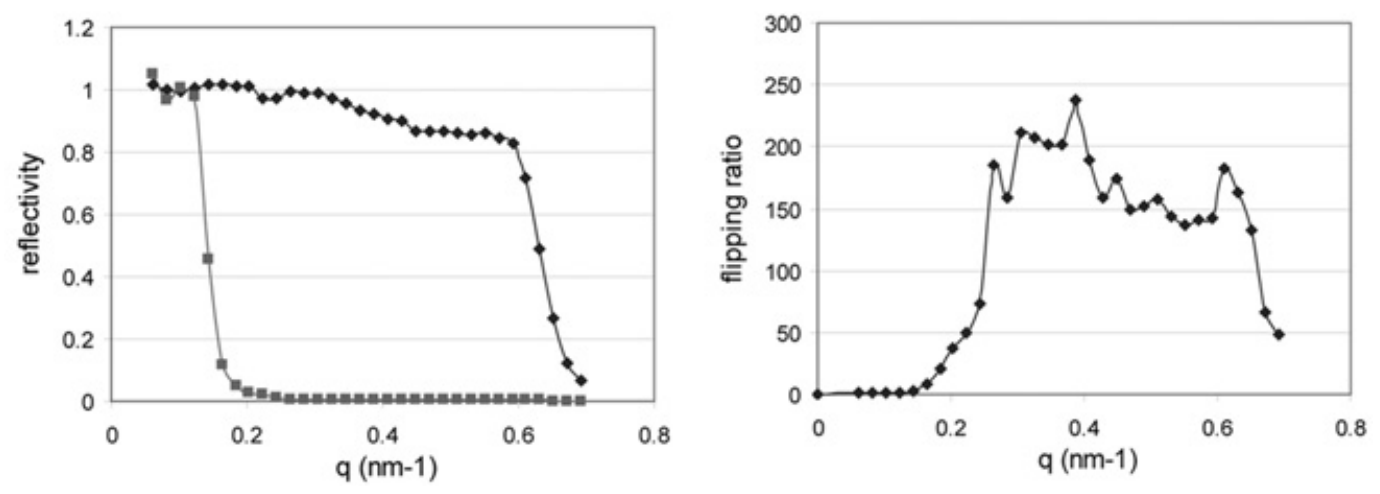

Figure 21. Réflectivité d'un super miroir polarisant.

\subsection{Multicouches magnétiques}

Nous avons présenté des éléments technologiques. De manière plus générale, la réflectivité de neutrons polarisés peut être utilisée pour étudier les configurations magnétiques dans un système multicouches. Cela permet :

- d'accéder à l'amplitude et à la direction de l'aimantation dans chaque couche, c'est-à-dire de déterminer les profils magnétiques en profondeur.

- de mesurer le moment magnétique de manière absolue en magnéton de Bohr.

La résolution en profondeur est de l'ordre de 2 à $3 \mathrm{~nm}$ dans les systèmes simples. La PNR est une technique de surface et n'est donc pas sensible aux contributions paramagnétiques et diamagnétiques du substrat. Il n'y a pas d'absorption. Il n'y a pas de paramètre phénoménologique. Les données sont naturellement normalisées.

\section{Couplage magnétique}

Dans un système de multicouches magnétiques où les couches magnétiques sont séparées par un espaceur magnétique, différents types de couplage peuvent apparaître. L'énergie de couplage magnétique peut être décrite phénoménologiquement par une énergie :

$$
E_{\text {coupling }}=-J_{1} \vec{S}_{1} \cdot \vec{S}_{2}-J_{2}\left(\vec{S}_{1} \cdot \vec{S}_{2}\right)^{2}
$$

Couplage d'échange dans les multicouches

Nous présentons l'exemple de multicouches $[\mathrm{Fe} / \mathrm{Si}]_{\mathrm{n}}$ produites par K. Fronc. Le système présenté est $\mathrm{GaAs} / /[\mathrm{Fe}(2.4 \mathrm{~nm}) \mathrm{Si}(1.2 \mathrm{~nm})]_{20}$. De la mesure de PNR, il est possible de déterminer qu'à température 


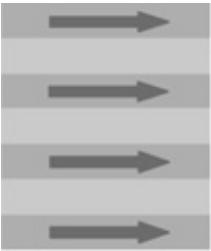

$\mathrm{J}_{1}>0$

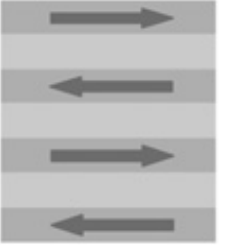

$\mathrm{J}_{1}<0$

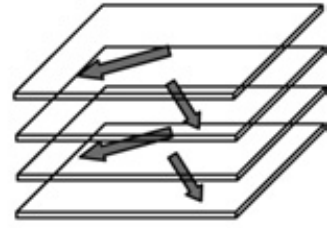

$\mathrm{J}_{1}>0$ and $\mathrm{J}_{2}<0$

Figure 22. (a) Couplage ferromagnétique ; (b) couplage antiferromagnétique (c) couplage quadratique.

ambiante, un ordre antiferromagnétique apparaît. Quant la température diminue, un ordre magnétique complexe apparaît. Sur la mesure de réflectivité (voir Figure 23), on peut observer différents détails. Le premier est le pic (001) de la structure du super-réseau qui correspond à la structure chimique du système. Le deuxième détail très important est le pic observé à la position $\left(\begin{array}{ll}0 & 0 \\ 1 / 2\end{array}\right)$. Ce pic est purement d'origine magnétique. Il indique qu'un couplage antiferromagnétique existe entre les couches de fer.

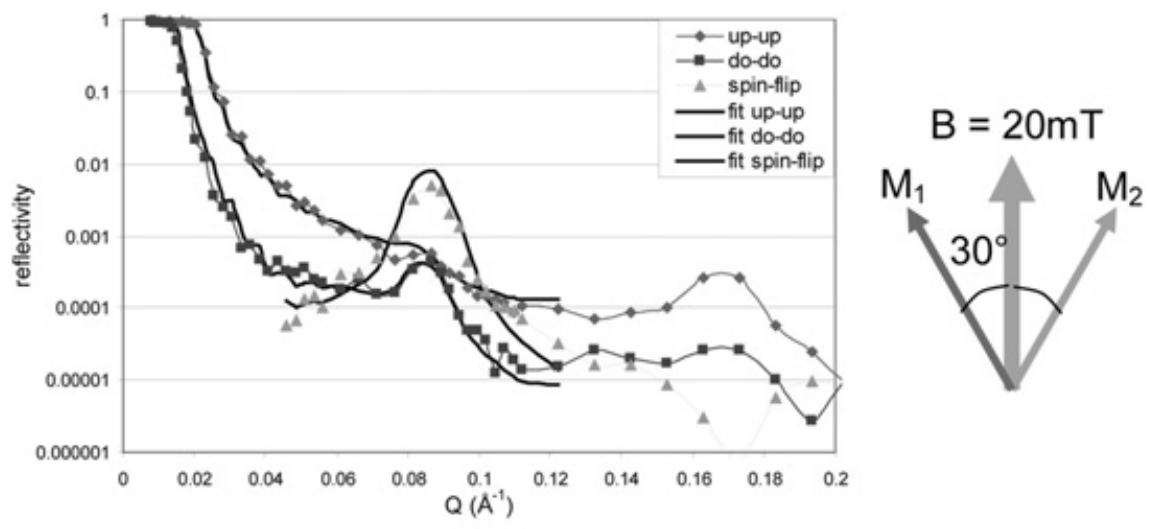

Figure 23. Réflectivités de $[\mathrm{Fe}(2.4) / \mathrm{Si}(1.2)]_{\mathrm{n}}$ mesurées à $5 \mathrm{~K}$.

Lorsque la température diminue en dessous de $200 \mathrm{~K}$, à la position du pic antiferromagnétique, un pic de spin-flip devient dominant. Cela signifie que le couplage entre les couches n'est plus purement antiferromagnétique. Un modèle numérique suggère que l'aimantation des couches de fer fait un angle de $30^{\circ}$ par rapport au champ magnétique appliqué.

\subsection{Couche mince magnétique métallique simple}

La PNR permet de sonder les systèmes très fins. Le comportement magnétique de $\mathrm{Fe} / \mathrm{Mn} / \mathrm{Fe}$ a été étudié. La structure typique de l'échantillon est présentée sur la Figure 24(a). La région active est formée par les couches $\mathrm{Fe} / \mathrm{Mn} / \mathrm{Fe}$. La couche d'argent est utilisée pour promouvoir la croissance épitaxiale des systèmes. La couche d'or est une simple couche de protection. Le système présenté est $\mathrm{Fe}_{0.5} \mathrm{Co}_{0.5} / \mathrm{Mn}\left(8 \mathrm{~A}^{\circ}\right) / \mathrm{Fe}_{0.5} \mathrm{Co}_{0.5}$. La spécificité de ce système est que les couplages magnétiques entre $\mathrm{Fe} / \mathrm{Mn}$ et $\mathrm{Co} / \mathrm{Mn}$ sont de signes opposés. Cela conduit un comportement magnétique complexe de la couche de manganèse.

Une première mesure a été réalisée dans un champ saturant (voir Figure 24(b)). Un modèle numérique des données montre que le moment magnétique dans le $\mathrm{Fe}_{0.5} \mathrm{Co}_{0.5}$ est de $2.4 \mu \mathrm{B}$ /atome (comme dans le 
matériau massif). Un moment magnétique net de $0.8 \mu \mathrm{B}$ /atome dans le Mn est aussi observé. Une telle aimantation induite a été théoriquement prédite pour les alliages FeCo. Dans des systèmes similaires sans cobalt, aucun moment magnétique n'est observé dans la couche de manganèse.
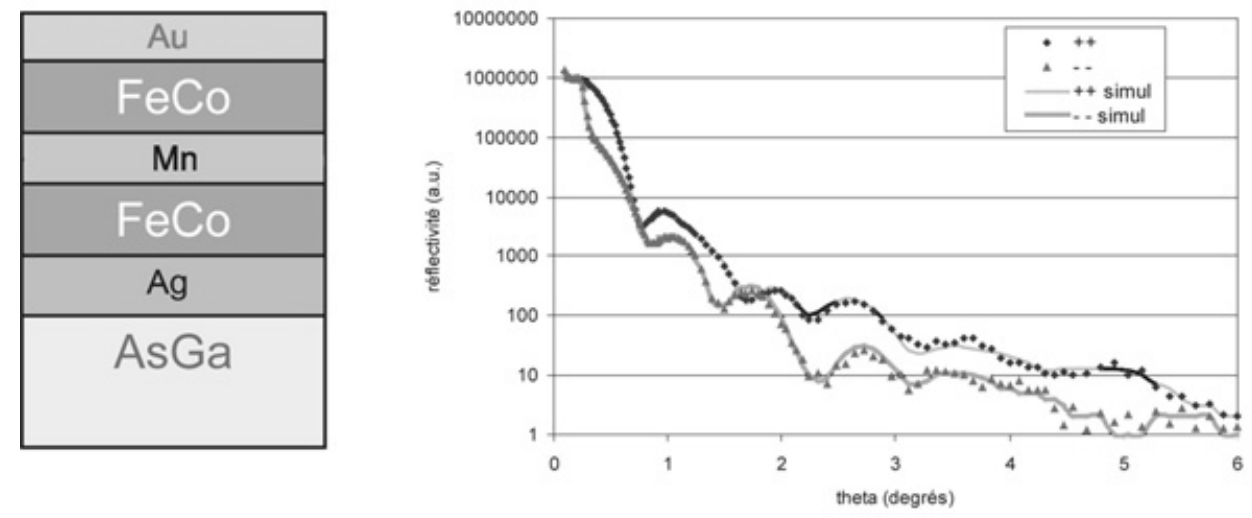

Figure 24. (a) Système tri-couche $\mathrm{Fe} / \mathrm{Mn} / \mathrm{Fe}$; (b) réflectivité dans l'état saturé.

Le champ magnétique appliqué a été diminué à $1.2 \mathrm{mT}$. La réflectivité a été mesurée à nouveau. Dans ces conditions, un fort signal de spin-flip est observé (voir Figure 25(a)). Les données de réflectivité ont été ajustées en laissant varier la direction de l'aimantation. Le meilleur fit est obtenu lorsque l'aimantation des couches fait un angle de $45^{\circ}$ par rapport au champ magnétique appliqué. Les deux aimantations de la multicouche font un angle de $90^{\circ}$, nous avons un couplage quadratique.
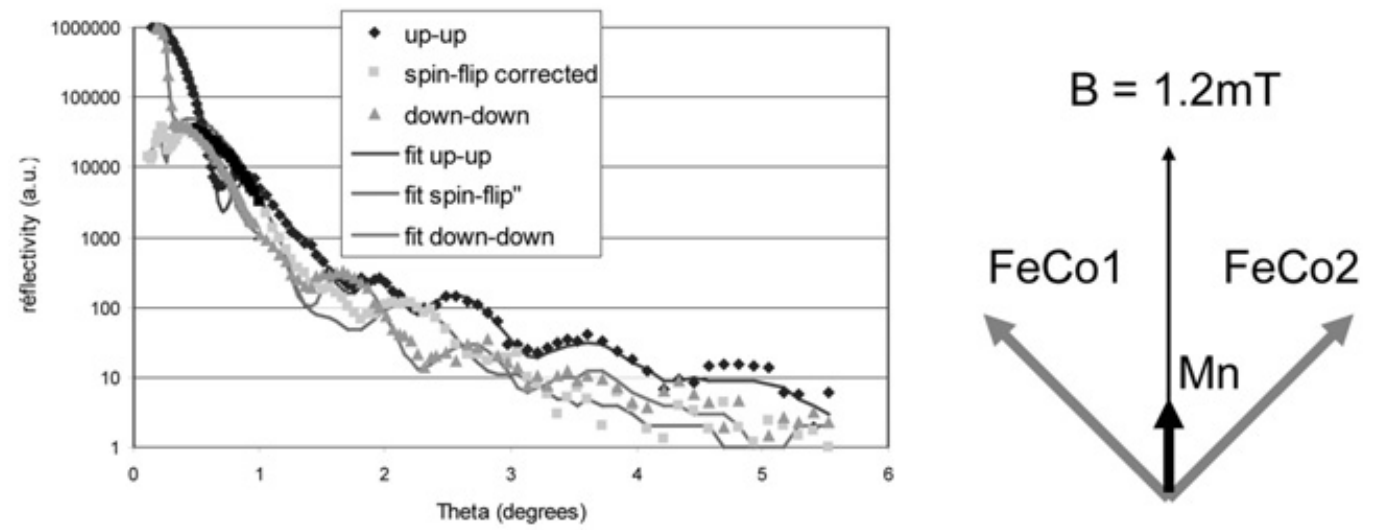

Figure 25. (a) Réflectivité dans l'état rémanant (b) configuration magnétique déduite du fit.

\subsection{Oxydes magnétiques}

Le premier exemple illustre l'utilisation du contraste magnétique pour mesurer la ségrégation chimique dans des hétéro-structures de manganites : $\left[\left(\mathrm{LaMnO}_{3}\right) \mathrm{a} /\left(\mathrm{SrMnO}_{3}\right) \mathrm{b}\right]_{\mathrm{n}}(\mathrm{avec} 8<\mathrm{a}<12 ; 4<\mathrm{b}<8)$. Ces super-réseaux sont déposés couche par couche afin de forcer un ordre cationique entre $\mathrm{La}$ et $\mathrm{Sr}$ et une ségrégation cationique entre $\mathrm{Mn}_{3+}$ et $\mathrm{Mn}_{4+}$. Le premier matériau est anti-ferromagnétique dans sa forme « bulk », le deuxième est ferromagnétique. L'objectif de la mesure était de voir si la ségrégation 
cationique $(\mathrm{La} / \mathrm{Sr}$ ) induisait effectivement un empilement (couche $\mathrm{AF} /$ couche $\mathrm{F}$ ). La réflectivité sur un de ces super-réseaux est présentée sur la Figure 26(a). Autour de l'angle $\theta=1.3^{\circ}$, on observe un pic de surstructure correspondant à la périodicité du système. Le contraste entre les 2 courbes de réflectivité « up » (rouge) et « down » (bleu) est caractéristique du profil d'aimantation dans la profondeur du film. Pour modéliser les courbes de réflectivité, il n'est nécessaire d'introduire qu'une faible modulation de l'aimantation dans le système (voir Figure 26(b)) : la ségrégation cationique ne conduit pas à une ségrégation magnétique franche. La modulation de l'aimantation n'est que de $25 \%$ entre les deux types de couches.
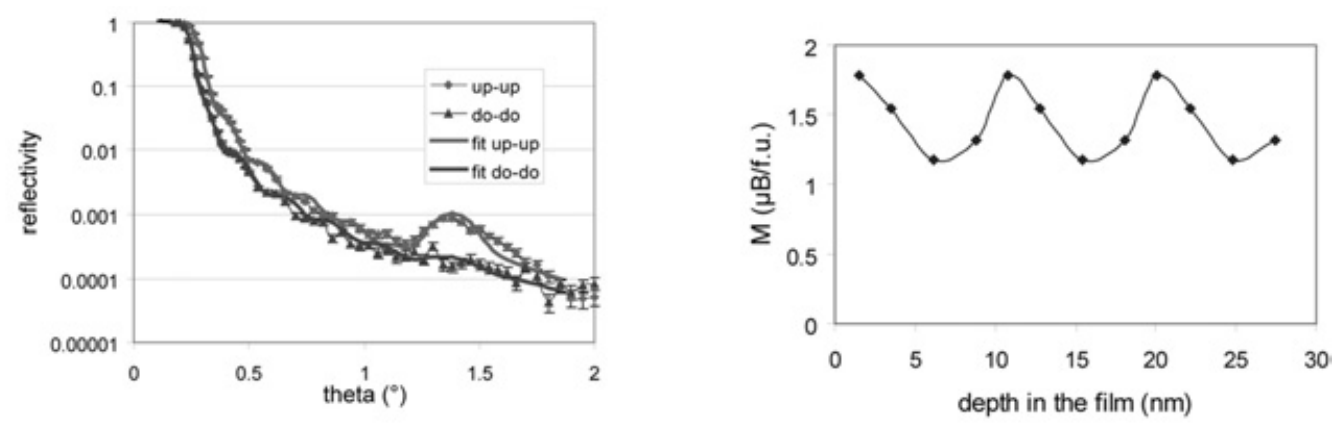

Figure 26. (a) Réflectivité sur un super-réseau $\left[\left(\mathrm{LaMnO}_{3}\right) \mathrm{a} /\left(\mathrm{SrMnO}_{3}\right) \mathrm{b}\right]_{\mathrm{n}}$. (b) profil d'indice optique magnétique dans le super-réseau.

Le deuxième exemple illustre l'utilisation du contraste magnétique afin de distinguer la structure dans une multicouche dont les composantes sont très proches : dans notre cas, des oxydes de fer $\mathrm{Fe}_{2} \mathrm{O}_{3}$

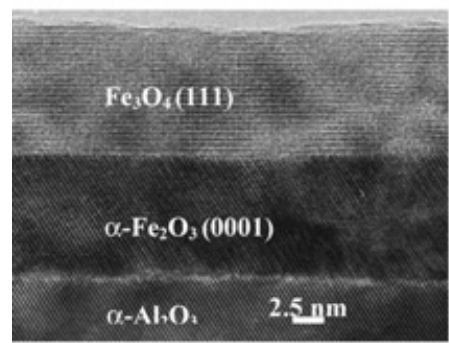

\section{Structure attendue}

\begin{tabular}{|c|l}
\hline $\mathrm{Fe} 3 \mathrm{O} 4$ & $\mathrm{M}=3.5 \mu \mathrm{B} / \mathrm{f} . u$. \\
${2 \mathrm{O} 3} }$ & $\mathrm{M}=0 \mu \mathrm{B} / \mathrm{f}$.u. \\
\hline $\mathrm{Al}_{2} \mathrm{O}_{3}$ & \\
\hline
\end{tabular}

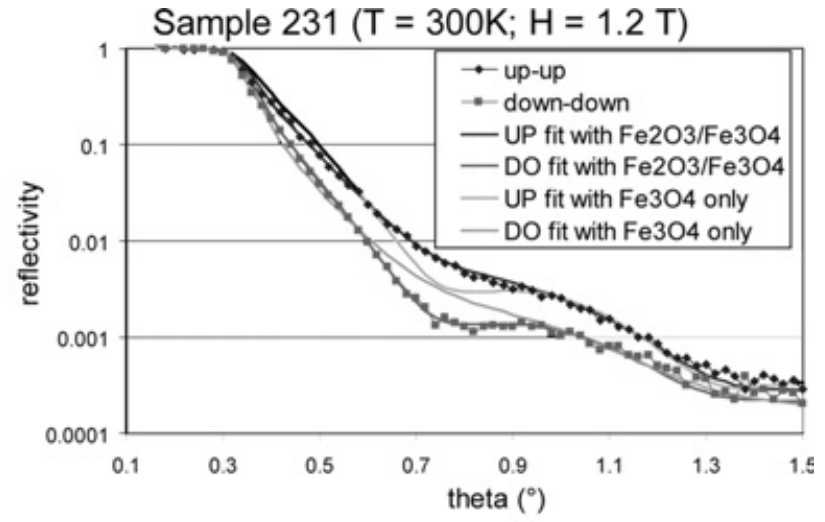

Structure obtenue

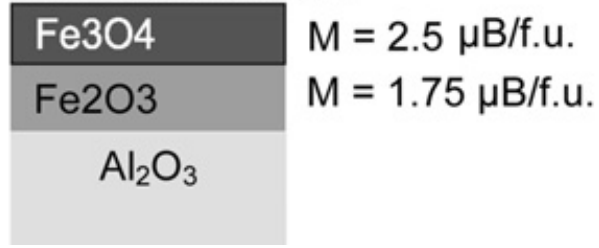

Figure 27. (gauche) bicouche $\mathrm{Fe}_{2} \mathrm{O}_{3} / \mathrm{Fe}_{3} \mathrm{O}_{4}$. (droite) réflectivité magnétique. (bas) profil magnétique attendu, profil magnétique ajusté. 
(anti-ferromagnétique) et $\mathrm{Fe}_{3} \mathrm{O}_{4}$ (magnétique). Ces oxydes présentent un intérêt dans la fabrication de jonctions tunnel magnétiques utilisant des courants polarisés en spin. La couche $\mathrm{AF}$ de $\mathrm{Fe}_{2} \mathrm{O}_{3}$ sert de couche de pinning à l'électrode de $\mathrm{Fe}_{3} \mathrm{O}_{4}$. La structure étudiée est présentée sur la Figure 27 gauche. La réflectivité de rayons $X$ ne permet pas de distinguer entre les 2 types de couches (la différence d'indice optique est de l'ordre de $1 \%$ ). Pour la réflectivité de neutrons, la différence d'indice optique est en principe très grande puisqu'un des matériaux est magnétique et pas l'autre. La Figure 27 droite présente la réflectivité sur ce système. L'empilement attendu est décrit sur la Figure 27 bas. Malheureusement, au lieu d'obtenir une ségrégation franche entre les deux couches, il y a eu une oxydoréduction entre les 2 couches : la couche de $\mathrm{Fe}_{2} \mathrm{O}_{3}$ a été réduite et est devenue magnétique ; la couche de $\mathrm{Fe}_{3} \mathrm{O}_{4}$ a été oxydée et a perdu de son magnétisme. Il est à noter que lors de la croissance, les diagrammes REED indiquaient la croissance des bonnes phases. Ce phénomène d'oxydoréduction a lieu sur des échelles de temps de l'ordre de la semaine.

\subsection{Manganites}

Nous allons nous intéresser aux composés de formule générale $\mathrm{A}_{1-x} \mathrm{~B}_{x} \mathrm{MnO}_{3}$. Les valences des différents ions sont : $\mathrm{A}^{3+}, \mathrm{B}^{2+}, \mathrm{Mn}^{3+/ 4+}, \mathrm{O}^{2-}$. La structure cristallographique est une structure pérovskite distordue (en raison de l'effet Jahn-Teller). L'environnement des Mn est octaédral (voir Figure 28).
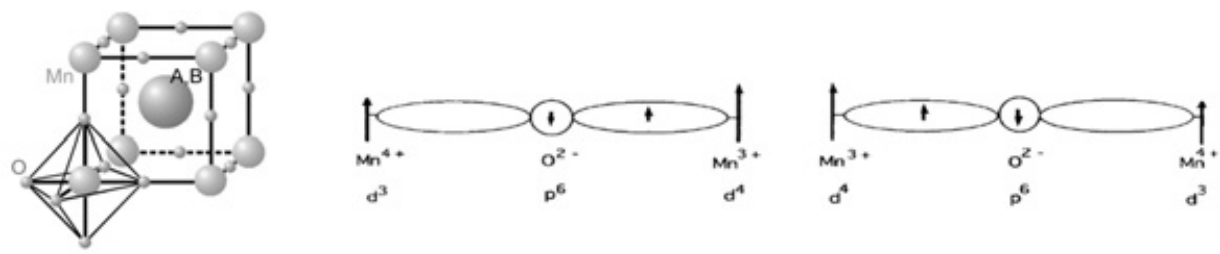

Figure 28. Structure typique d'une pérovskite avec $\mathrm{A}=\mathrm{La}$ et terres rares ; $\mathrm{B}=\mathrm{Ca}, \mathrm{Ba}, \mathrm{Sr}, \mathrm{Pb} \ldots$

La valence du Mn (3+ ou 4+) influe sur le dopage électronique. Les interactions magnétiques sont de deux types dans ces pérovskites :

- Super-échange (entre $\mathrm{Mn}^{4+}$ et $\mathrm{Mn}^{4+}$ )

Dans ce cas, les charges sont localisées et l'échange dépend essentiellement des configurations des orbitales. Ce type d'échange est le plus souvent anti-ferromagnétique. Il suit les règles de Goodenough-Kanamori.

- Double-échange (entre $\mathrm{Mn}^{3+}$ et $\mathrm{Mn}^{4+}$ ) (voir Figure 28)

Dans le cas d'une liaison $\mathrm{Mn}^{3+}-\mathrm{Mn}^{4+}$, il existe 2 états dégénérés. Un électron peut être échangé entre les deux ions Mn avec une mémoire de spin. Cela conduit à un couplage ferromagnétique et à un comportement métallique du composé. Dans ces composés, la bande de conduction électronique est polarisée à $100 \%$.

Nous avons réalisé des mesures de réflectivité de neutrons sur des couches minces de ces composés. Dans le cas des couches minces, le cycle d'hystérésis (voir Figure 29) présente une partie avec une très faible coercivité à laquelle se superpose une contribution qui nécessite $0.3 T$ pour être saturée. Cela suggère que les films de manganites ne sont pas homogènes et qu'ils sont constitués de plusieurs phases ayant des coercivités différentes. Nous avons utilisé la réflectivité de neutrons pour sonder l'aimantation de ces couches en fonction de la profondeur pour différentes températures et valeurs de champ. La Figure 30 présente la réflectivité d'une couche de $\mathrm{La}_{0.7} \mathrm{Sr}_{0.3} \mathrm{MnO}_{3}$ de $16 \mathrm{~nm}$. Afin de modéliser quantitativement les données, il a été nécessaire d'introduire un modèle qui tient compte d'une aimantation différente aux interfaces. La modélisation de la couche mince par une couche uniforme (Figure 31) ne permet pas de rendre compte des réflectivités mesurées. Nous avons donc considéré un système à trois 
couches avec des aimantations $M_{1}, M_{2}$ et $M_{3}$ dans l'épaisseur du film. La Figure 31 présente les variations des aimantations $M_{1}, M_{2}$ et $M_{3}$ en fonction de la température. On constate que l'aimantation aux interfaces est réduite de 25 à $30 \%$.

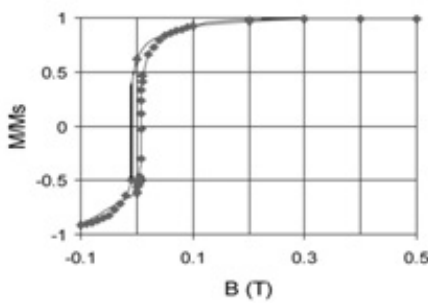

Figure 29. Cycle d'hystérésis d'un film de $40 \mathrm{~nm}$ de $\mathrm{La}_{0.7} \mathrm{Sr}_{0.3} \mathrm{MnO}_{3}$ déposé sur un substrat de $\mathrm{SrTiO}_{3}$.
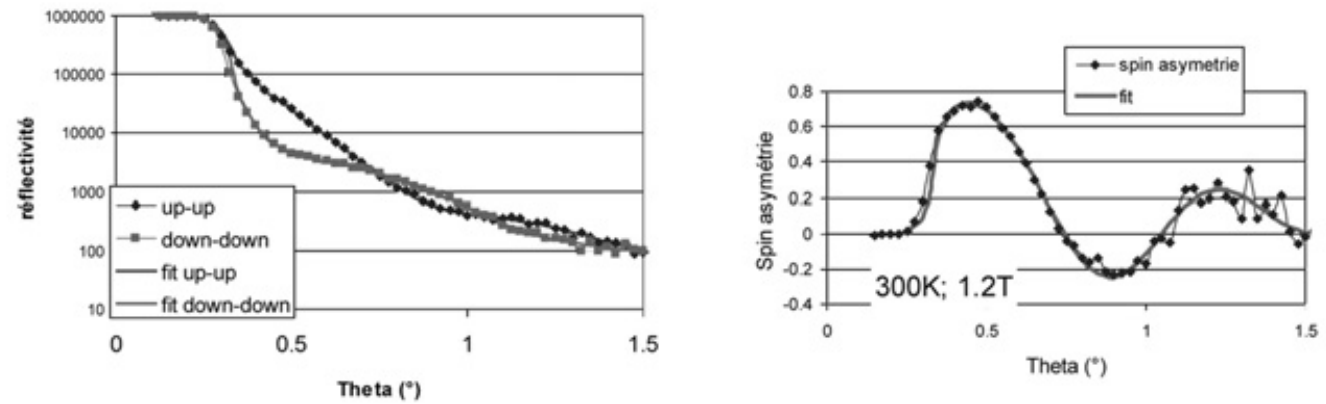

Figure 30. Réflectivité d'un film de $\mathrm{La}_{0.7} \mathrm{Sr}_{0.3} \mathrm{MnO}_{3}(16 \mathrm{~nm})$ déposé sur $\mathrm{SrTiO}_{3}$. (gauche) le rapport d'asymétrie.
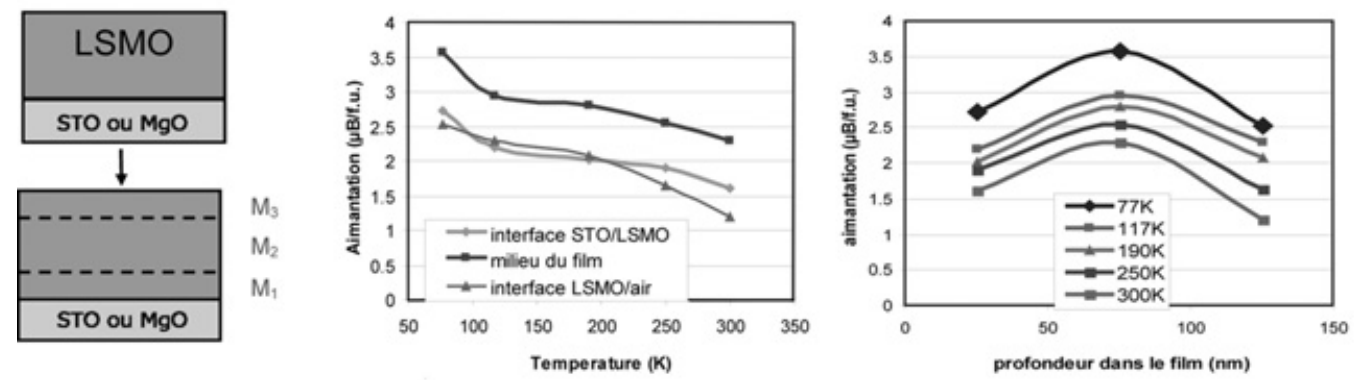

Figure 31. (gauche) modélisation du système : (haut) système parfait ; (bas) modèle plus réaliste. Variations des aimantations $M_{1}, M_{2}$ et $M_{3}$ pour le système $\mathrm{LSMO}(16 \mathrm{~nm}) / / \mathrm{STO}$.

\subsection{Couplage d'échange}

\section{Couplage d'échange}

Au niveau d'une interface entre un matériau ferro (F) et un matériau anti-ferromagnétique (AF), il existe en général un couplage magnétique. Ce couplage magnétique a pour effet d'introduire un décalage dans le cycle d'hystérésis. Il existe différents modèles pour expliquer cet effet : 
- Modèle de l'état en domaines [Nowak et al, JMMM 240, 243 (2002) ; PRB 66, 014430 (2002)] Le comportement réversible des moments de l'AF non compensés à l'interface donne lieu à la coercivité.

L'aimantation réversible de l'AF suit celle de la couche ferromagnétique. Les moments non compensés de l'AF gelés à l'interface (défauts, parois de domaines) donnent lieu à un renversement irréversible de l'aimantation de l'AF et au champ d'échange.

- Modèle de la paroi de domaine incomplète dans le ferro [M. Kiwi, JMMM 234 584-595 (2001)]

Dans le cas d'un fort couplage et d'une grande anisotropie dans l'AF : il doit y avoir formation de parois de domaine dans le ferromagnétique. Le système idéal pour étudier cela est $\mathrm{FeF}_{2} / \mathrm{Fe}$.

(a)

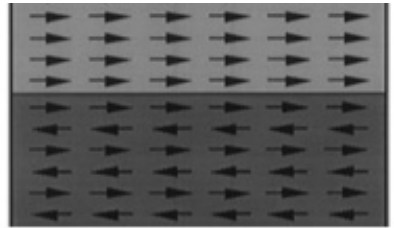

(b)

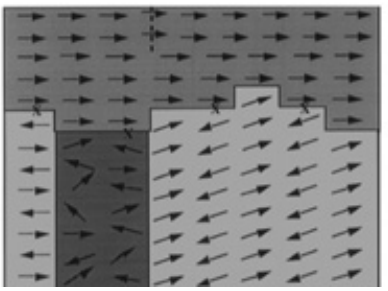

$\mathbf{F}$

\section{FI AF}

AF (c)

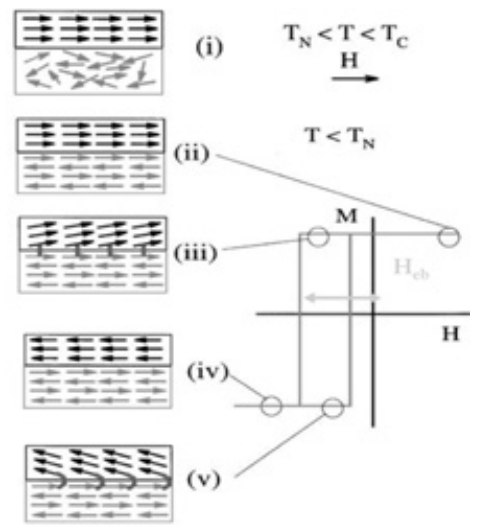

Figure 32. (a) Couplage magnétique entre une interface Ferro / AF. (b) Les vrais échantillons sont loin du cas idéal. (i) Il y a des interactions d'échanges aléatoires (rugosité d'interface), (ii) il $y$ a des parois de domaines dans l'AF : parois de domaines $\perp$ (antiphases) ou// (twist) à l'interface, (iii) présence de spin non compensés (défauts dans l'AF, joints de grains, mélange $\cdots$... (c) Retournement de l'aimantation dans un système F/AF.

Dans le cas du système $\mathrm{CoO} / \mathrm{Co} / / \mathrm{Si}$, on peut observer que le mécanisme de renversement n'est pas le même à l'aller et au retour du cycle d'hystérésis (voir figure ci-dessous).
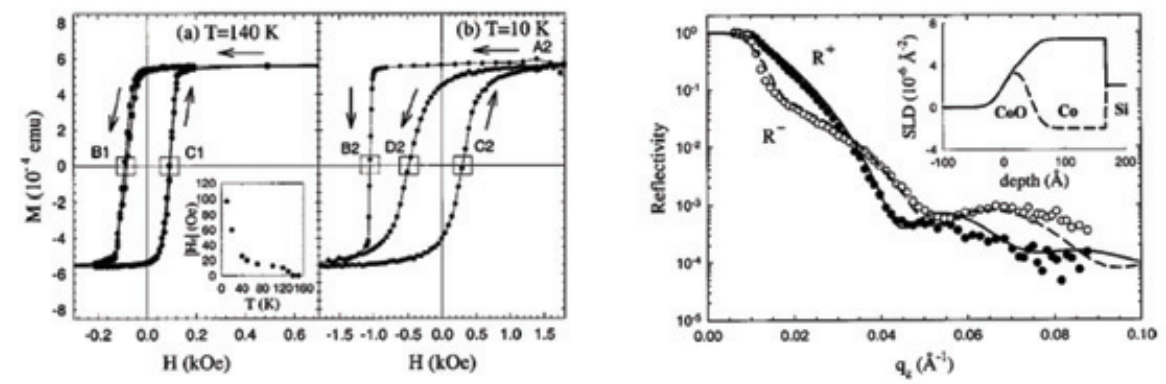

Figure 33. (a) renversement de l'aimantation dans le système $\mathrm{CoO} / \mathrm{Co} / / \mathrm{Si}$ [W.-T. Lee et al., PRB65, 224417 (2002)]. (b) PNR à saturation pour obtenir les épaisseurs et la rugosité du système $(\mathrm{Co}=13 \mathrm{~nm}, \mathrm{CoO}=4.5 \mathrm{~nm})$. 


\section{Utilisation de la PNR}

A partir des mesures de PNR, il est possible de déterminer la direction de l'aimantation. Soit $R(\phi)$ la réflectivité dans le cas où la polarisation des neutrons fait un angle $\phi$ avec l'aimantation. On peut montrer que si l'aimantation est constante dans l'épaisseur du film (en direction et en amplitude) :

$$
\begin{aligned}
\frac{R^{++}(\phi)-R^{--}(\phi)}{R_{s}^{++}\left(0^{\circ}\right)-R_{s}^{--}\left(0^{\circ}\right)} & =\cos (\phi) \\
\frac{R^{-+}(\phi)}{R_{s}^{-+}\left(90^{\circ}\right)} & =\sin ^{2}(\phi)
\end{aligned}
$$

Mais il est possible d'obtenir plus d'informations sur l'état micromagnétique de la couche ferromagnétique. Dans le cas de multidomaines, la réflectivité dépend de la taille des domaines. Quand les domaines sont petits, on mesure la valeur de l'aimantation $\langle M\rangle$. Quand les domaines sont grands, les intensités réfléchies par les différents domaines s'additionnent de manière incohérente. Il est donc possible d'obtenir des informations sur la moyenne aux moindres carrés de la dispersion de l'orientation des domaines $\chi^{2}$. Cette relation est valable quel que soit le vecteur de diffusion mais à la condition que les domaines magnétiques soient suffisamment grands

$$
\begin{aligned}
\chi^{2} & =\left\langle\cos ^{2} \varphi\right\rangle-\langle\cos \varphi\rangle^{2} \\
& =\left\{1-\frac{R^{-+}(\varphi)}{R_{s}^{-+}\left(90^{\circ}\right)}\right\}-\left\{\frac{R^{++}(\varphi)-R^{--}(\varphi)}{R_{s}^{++}\left(0^{\circ}\right)-R_{s}^{--}\left(0^{\circ}\right)}\right\}^{2}
\end{aligned}
$$
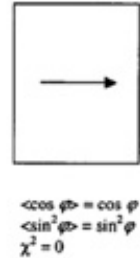

(a)
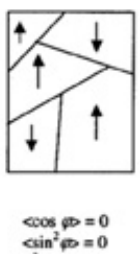
$x^{2}=1$
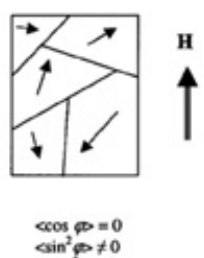

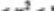

Figure 34. (i) Dans le cas d'une aimantation homogène, $\chi^{2}=0$. (ii) dans le cas de grands domaines orientés AF, $\chi^{2}=1$. (iii) dans le cas intermédiaire $0<\chi^{2}<1$.

Dans le cas du système $\mathrm{CoO} / \mathrm{Co} / / \mathrm{Si}$, les résultats des mesures de PNR sont présentés sur la Figure 35.
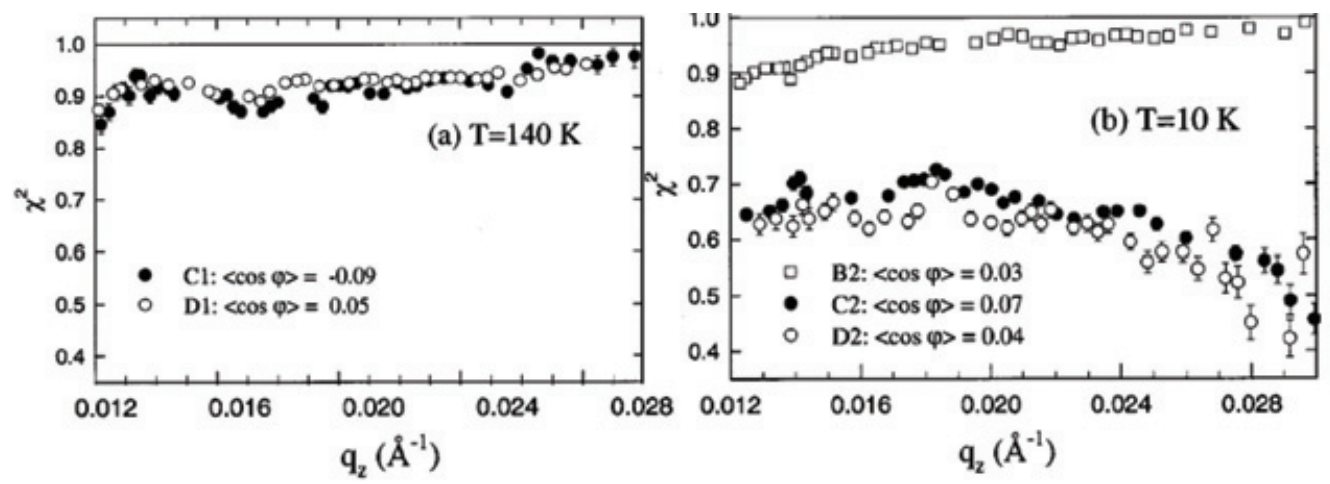

Figure 35. Tracé de aux point C1, D1, B2, C2, D2 des cycles d'hystérésis (voir Figure 33).

On peut déduire de ces mesures que pour les états B1,C1 et B2, il y a un retournement uniaxial des domaines (Figure 34, cas b). Dans les cas C2 et D2, il y a une dispersion considérable des directions des aimantations (Figure 34, cas c). Après "training", le couplage d'échange des différents domaines cristallographiques de $\mathrm{CoO}$ est aléatoire. 


\subsection{Exemple du système $\mathrm{MgO} / / \mathrm{FeF}_{2} / \mathrm{Fe}$}

Nous présentons des mesures sur le système $\mathrm{MgO} / \mathrm{FeF}_{2}(90 \mathrm{~nm}) / \mathrm{Fe}(75 \mathrm{~nm}) / \mathrm{Al}(7 \mathrm{~nm})$.

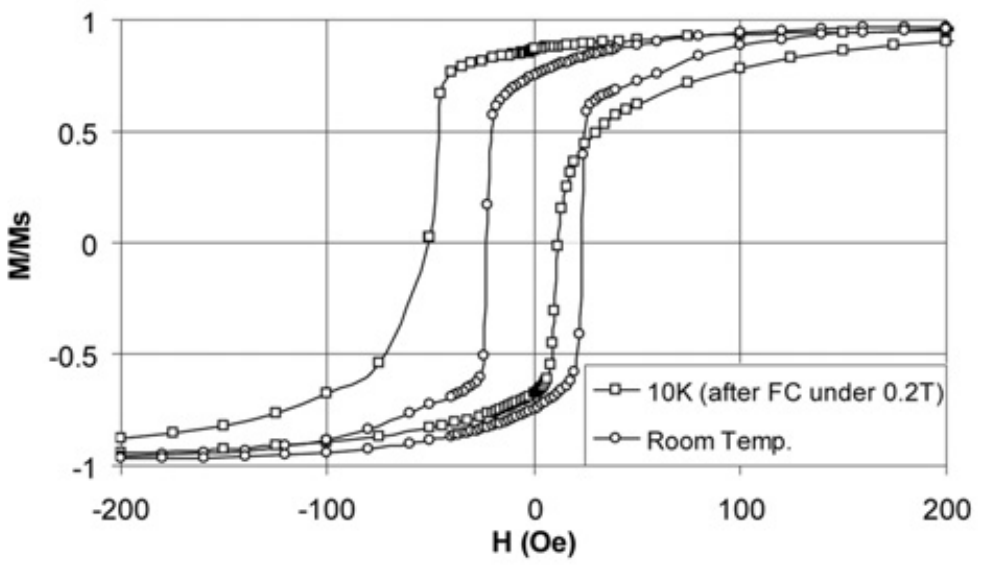

Figure 36. Cycle d'hystérésis (i) à $300 \mathrm{~K}, \mathrm{Hc}=22 \mathrm{Oe}$, rémanence $=0.8 \mathrm{Ms}$. (ii) à $10 \mathrm{~K}$ après $\mathrm{FC}(0.2 \mathrm{~T})$ : $\mathrm{He}=-14 \mathrm{Oe}, \mathrm{Hc}=31 \mathrm{Oe}$, rémanence $=0.87 \mathrm{Ms}$.

Dans le cas de films épais, le couplage d'échange est plus faible que dans le cas des films très minces $(20 \mathrm{~nm})$. Le retournement est aussi plus doux que dans le cas des structures plus fines.

La Figure 37 présente les mesures de réflectivité de neutrons polarisés réalisées sur le spectromètre PRISM au LLB. Les quatre sections efficaces sont mesurées $R^{++}, R^{--}, R^{+-}, R^{-+}$. La polarisation des neutrons est parallèle au champ magnétique appliqué (dans le plan de l'échantillon) ainsi qu'à l'aimantation du film. La composante de l'aimantation perpendiculaire au spin du neutron, c'est à dire au champ magnétique appliqué, est responsable pour les processus de spin-flip $R^{+-}$et $R^{-+}$. Ces intensités apparaissent donc uniquement lorsque l'aimantation de l'échantillon n'est pas totalement alignée avec le spin des neutrons incidents.

La Figure 37(a) présente la réflectivité à $300 \mathrm{~K}$ sous 1 T. A l'ambiante, ce fort champ est largement suffisant pour saturer totalement l'échantillon et le signal de spin flip est donc absent. Pour modéliser le système et rendre compte des réflexions, il est nécessaire d'introduire un profil en épaisseur composé de couches de $4 \mathrm{~nm}$ à l'interface $\left(\mathrm{FeF}_{2} / \mathrm{Fe}\right.$ et $\left.\mathrm{Fe} / \mathrm{Al}\right)$ et un moment réduit. L'épaisseur totale de la couche de fer est de $75 \mathrm{~nm}$.
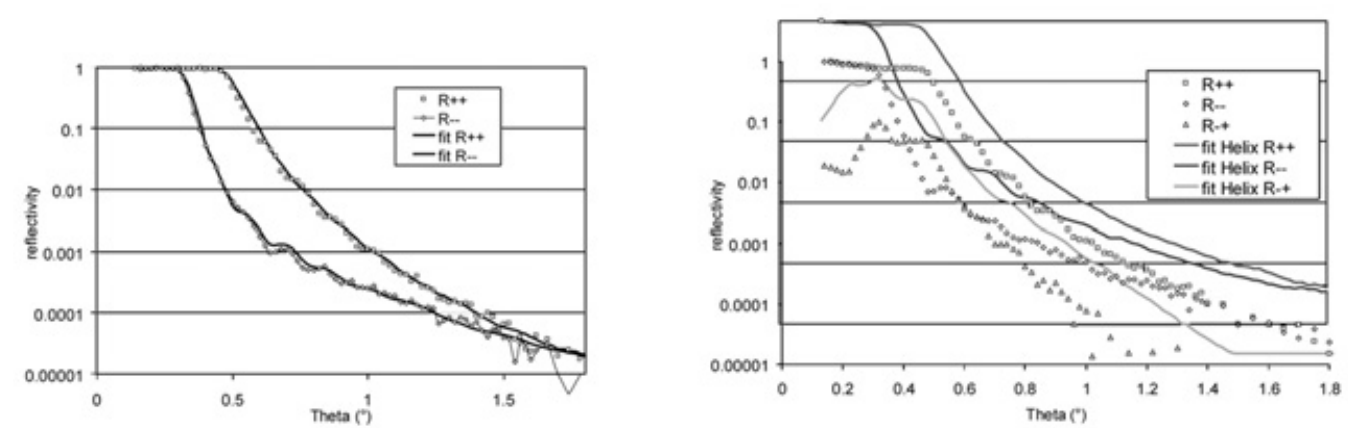

Figure 37. (a) Mesure à $300 \mathrm{~K}$ sous $1 \mathrm{~T}$ afin d'obtenir les paramètres du système (SLD, aimantation, rugosité). (b) mesure à $76 \mathrm{G} ; 10 \mathrm{~K}$ (juste avant le retournement). 
La Figure 37(b) présente la mesure à $10 \mathrm{~K}$ après refroidissement sous $105 \mathrm{G}$. La réflectivité a ensuite été mesurée à $-13 \mathrm{Oe},-50$ Oe et -76 Oe pour analyser le retournement de la partie gauche du cycle d'hystérésis. Les data présentées correspondent à la mesure à -76 Oe (juste avant le retournement). La présence d'un signal significatif de spin flip démontre la présence d'une composante de l'aimantation perpendiculaire au champ (et donc au spin des neutrons). Le coeur du problème consiste à analyser précisément le profil d'aimantation. La première hypothèse est de considérer une couche homogène qui ferait un angle non nul avec le champ extérieur. En utilisant les paramètres tirés de la mesure à saturation, le meilleur fit possible est représenté en bleu et correspond à une aimantation faisant un angle de $25^{\circ}$ par rapport au champ magnétique appliqué. Le fit obtenu n'est pas très satisfaisant, en particulier en ce qui concerne l'évaluation de $R^{--}$. Il apparait donc qu'un paramètre supplémentaire soit requis pour reproduire l'ensemble des trois courbes mesurées. En rouge, nous considérons la même rotation globale de l'aimantation mais en superposant une structure en hélice. Le fit est clairement meilleur, mais ce résultat est attendu vu l'ajout d'un paramètre de fit supplémentaire. La question est donc de trancher sur la pertinence physique de ce qui doit être rajoutéé pour expliquer les mesures. Discutons donc des différents états possibles du retournement.

Premières conclusions à partir des data :

La question de l'état multidomaines Vs monodomaine

Pour une configuration en polydomaines, la courbe de réflectivité peut être affectée de plusieurs manières. Si la taille des domaines est plus grande que la longueur de cohérence des neutrons, la réflectivité est la somme pondérée des spectres $R^{++}$et $R^{--}$. Il est intéressant de noter que pour $=0.48^{\circ}, R^{++} / R^{--}$est supérieur à 50, ce qui implique une proportion de grands domaines inférieure à $2 \%$. Si les domaines sont très petits, la réflectivité est donnée par la moyenne des amplitudes des aimantations auquel s'ajoute un petit terme de spin-flip issu du moment moyen perpendiculaire au spin des neutrons incidents. Cela n'est pas cohérent avec les data. On en déduit que nous avons un état monodomaine.

La question de la structure en hélice

L'ensemble des spectres à différentes températures peut être raisonnablement modélisé par une rotation cohérente de l'aimantation. On observe une amélioration des ajustements si on autorise une structure de type hélice dans l'épaisseur de la couche de fer. Cependant, cela suppose un plus grand nombre de paramètres et il est donc difficile de conclure de manière non ambiguë.

Il existe cependant une manière plus directe de montrer que l'aimantation n'est pas homogène dans l'épaisseur du film. Pour ceci, on se rappellera que la quantité $\left(R^{++}-R^{--}\right) /\left(R^{++}-R^{--}\right)$sat est égale au cosinus de l'angle entre l'aimantation et le spin des neutrons $\cos (\varphi)$ (voir Eq. 39). Dans le cas d'une aimantation homogène, $\cos (\varphi)$ est constant quelle que soit la valeur du vecteur de diffusion. Il est toutefois intéressant de noter que ce vecteur est précisément relié à une taille typique sondée, et que l'intensité des neutrons correspond grossièrement à une moyenne de l'amplitude des ondes diffusées à une échelle plus petite que celle donnée par le vecteur d'onde et que ce sont les intensités qui s'ajoutent à une échelle plus grande. Ainsi, toute variation de l'angle en profondeur doit affecter la valeur de ce cosinus mesurée à une échelle en q correspondant à des tailles plus petites que l'échelle caractéristique de la variation de M. La Figure 38 présente la quantité $\cos (\varphi)$ dans le cas de notre système en fonction de l'angle (c'est-à-dire du vecteur de diffusion). Les points expérimentaux ne sont pas sur une ligne horizontale et deux échelles peuvent être extraites à première vue. La fréquence d'oscillation rapide correspond à l'épaisseur totale de la couche de Fe. La courbure générale indique la présence d'une structure en hélice. Le tilt global est de l'ordre de $25^{\circ}$; l'aimantation est non colinéaire dans l'épaisseur de la couche. A partir du traitement quantitatif des fits : l'amplitude des variations angulaires de l'aimantation est de l'ordre de $30^{\circ}$ sur une épaisseur de $20 \mathrm{~nm}$ à partir de l'interface.

En utilisant la réflectivité de neutrons polarisés, il est donc possible d'obtenir des informations sur la distribution d'aimantation dans l'épaisseur d'un film mince, non seulement en amplitude (réduite ou augmentée aux interfaces), mais aussi en direction (structures en hélices). Dans le cas de l'interaction 


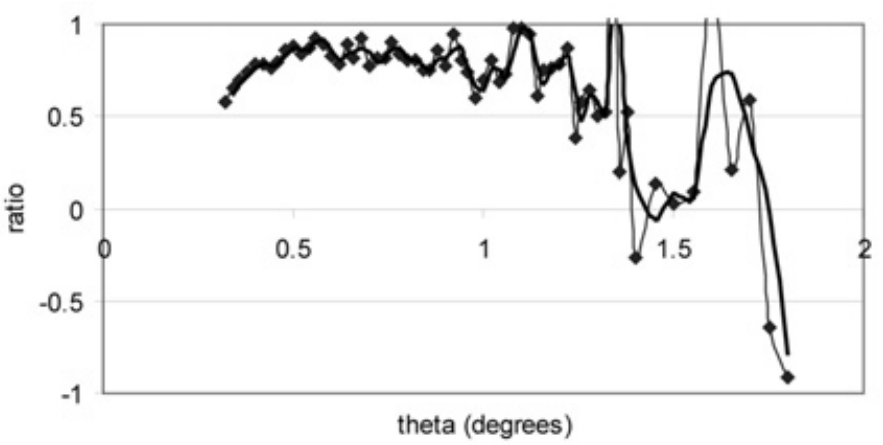

Figure 38. Asymétrie normalisée par l'asymétrie à saturation.

d'échange dans le système $\mathrm{FeF}_{2} / \mathrm{Fe}$, nous avons pu mettre en évidence l'existence d'une structure en éventail sur une épaisseur d'une vingtaine de nanomètres de l'ordre de $30^{\circ}$ lors du retournement de l'aimantation.

\section{CONCLUSION}

Nous avons présenté différentes possibilités d'utilisation de la réflectivité de neutrons polarisés pour l'étude de films minces. Les études étaient limitées à la caractérisation de profils magnétiques en profondeur dans les multicouches. Il est possible d'étendre ces études pour caractériser les structures planaires dans des systèmes multicouches. Cela sera présenté par E. Kentzinger dans le chapitre qui discute de la diffusion hors spéculaire et de la diffusion en incidence rasante (Grazing Incidence SANS). 